Pure and Applied Mathematics Quarterly

Volume 2, Number 4

(Special Issue: In honor of

Robert MacPherson, Part 2 of 3)

$1149-1186,2006$

\title{
Remarks on the Combinatorial Intersection Cohomology of Fans
}

\author{
Tom Braden \\ For Bob MacPherson on his sixtieth birthday
}

\begin{abstract}
We review the theory of combinatorial intersection cohomology of fans developed by Barthel-Brasselet-Fieseler-Kaup, Bressler-Lunts, and Karu. This theory gives a substitute for the intersection cohomology of toric varieties which has all the expected formal properties but makes sense even for non-rational fans, which do not define a toric variety. As a result, a number of interesting results on the toric $g$ and $h$ polynomials have been extended from rational polytopes to general polytopes. We present explicit complexes computing the combinatorial IH in degrees one and two; the degree two complex gives the rigidity complex previously used by Kalai to study $g_{2}$. We present several new results which follow from these methods, as well as previously unpublished proofs of Kalai that $g_{k}(P)=0$ implies $g_{k}\left(P^{*}\right)=0$ and $g_{k+1}(P)=0$.
\end{abstract}

For a $d$-dimensional convex polytope $P$, Stanley [St2] defined a polynomial invariant $h(P, t)=\sum_{k=0}^{d} h_{k}(P) t^{k}$ of its face lattice, which is usually called the "generalized" or "toric" $h$-polynomial of $P$. It is "generalized" in that it extends a previous definition from simplicial polytopes to general polytopes, while the adjective "toric" refers to the fact that if $P$ is a rational polytope (meaning that all its vertices have all coordinates in $\mathbb{Q}$ ), then the coefficients of $h(P, t)$ are intersection cohomology Betti numbers of an associated projective toric variety $X_{P}$ :

$$
h_{k}(P)=\operatorname{dim}_{\mathbb{R}} I H^{2 k}\left(X_{P} ; \mathbb{R}\right) .
$$

In fact, these Betti numbers had been computed independently by several people, including Robert MacPherson, and these calculations inspired Stanley's definition

Received November 20, 2005.

The author was supported in part by NSF grant DMS-0201823. 
(although the first published proofs [DL, Fies] of (1) did not appear until several years after Stanley's definition of the $h$-polynomial).

This connection between the topology of toric varieties and combinatorics of polytopes was then used to prove a number of interesting relations among these invariants [Bay1, BM, St3]; these results were therefore known only for rational polytopes. It was believed, however, that they should hold for general polytopes, and in a few cases (simplicial polytopes [McM2] and low degree terms [Kal1]) non-toric proofs were found.

The recent papers [BBFK2, BrL1, Kar1] have settled this question, by defining groups which can substitute for intersection cohomology in (1), but which are defined whether or not $P$ is rational. In fact, they accomplish more: they define a theory of sheaves purely in terms of the linear structure of $P$ which completely captures the structure of torus-equivariant constructible sheaves on the toric variety $X_{P}$ when $P$ is rational. The maps and other relations between intersection cohomology groups which were used to prove identities and inequalities among the $h$-numbers [BM, St3] have direct analogues in the new theory. Moreover, the deep theorems from algebraic geometry that were used to prove (1) - the Decomposition Theorem and the Hard Lefschetz Theorem - now have proofs in this combinatorial setting. The result is a powerful, self-contained theory which can answer many questions about the combinatorics of convex polytopes.

This paper is meant as a guided introduction to the theory of combinatorial intersection cohomology and some of its applications. In Section 1 we describe the toric $g$ - and $h$-numbers and the main results about them. In Section 2 we review the construction of the combinatorial intersection cohomology sheaves and their main properties. We describe what their graded pieces look like in degrees up to two; in degree two we recover a chain complex related to rigidity of frameworks which Kalai used as a substitute for the intersection cohomology group $I H^{4}\left(X_{P} ; \mathbb{R}\right)$.

In Section 3 we present two applications of a result of [BBFK2] on "quasiconvex" fans which have not appeared before. First, we answer a question of Stanley [St2] regarding whether local contributions to $h(P, t)$ at a facet have nonnegative coefficients. Second, we present an inequality which generalizes Kalai's monotonicity with $t$ specialized to 1 . In Section 4 we describe how Stanley's convolution identity which relates the $g$-numbers of $P$ and the polar polytope $P^{*}$ can be "lifted" to exact sequences, and relate this to the Koszul duality constructed in [BL]. Finally, in an appendix we present Kalai's proofs of two interesting applications of monotonicity.

Although our discussion is developed entirely in the setting of convex geometry, in many places we point out the connections with the topology of toric varieties. Although this is no longer necessary to understand the proofs, we hope that 
the reader will appreciate seeing these connections as a way to motivate the constructions and suggest new applications.

Thanks are due to Gottfried Barthel, Jean-Paul Brasselet, Karl-Heinz Fieseler, Ludger Kaup, Valery Lunts, and Gil Kalai for many interesting conversations over the years, to Chris McDaniel for helpful comments on a draft of this article, and to Bob MacPherson for introducing me to the beautiful interplay between geometry and combinatorics.

\section{The $g$ - AND $h$-POLYNOMIALS}

1.1. Simplicial polytopes. We start with a discussion of the situation for simplicial polytopes. When $P$ is simplicial, the $h$-polynomial $h(P, t)=\sum_{k=0}^{d} h_{k}(P) t^{k}$ is defined by

$$
h(P, t)=(t-1)^{d}+f_{0}(t-1)^{d-1}+\cdots+f_{d-1},
$$

where the face number $f_{k}=f_{k}(P)$ is the number of $k$-dimensional faces of $P$. The transformation taking $\left\{f_{k}\right\}$ to $\left\{h_{k}\right\}$ is invertible, so that the $h$-numbers determine the face numbers.

The $h$-numbers are not independent - they satisfy the Dehn-Sommerville relations:

$$
h_{k}(P)=h_{d-k}(P) \text { for all } 1 \leq k \leq d .
$$

As a result, if we define an auxiliary polynomial $g(P, t)=\sum_{k=0}^{\lfloor d / 2\rfloor} g_{k}(P) t^{k}$ by

$$
g_{k}(P)=h_{k}(P)-h_{k-1}(P), 0 \leq k \leq d / 2,
$$

where we put $h_{-1}(P)=0$, then $g(P, t)$ contains all the information of $h(P, t)$, and thus all the information about the face numbers.

The study of these invariants culminated in the proof of the " $g$-theorem", which was originally conjectured by McMullen:

Theorem 1.1 ([BiL, St1]). A sequence $f_{0}, \ldots, f_{d-1} \in \mathbb{Z}_{\geq 0}$ are the face numbers of a d-dimensional simplicial polytope if and only if

- the Dehn-Sommerville relations $h_{k}=h_{d-k}$ hold,

- $g_{k}(P) \geq 0$ for $0 \leq k \leq d / 2$, and

- there exists a graded ring $H=\oplus_{i \geq 0} H_{i}$, with $H_{0}=\mathbb{R}$ and generated by $H_{1}$, for which $g_{i}=\operatorname{dim}_{\mathbb{R}} H_{i}$.

Sequences satisfying the last condition are known as "M-sequences". Being an M-sequence is equivalent to a set of non-linear inequalities. For instance, $1, g_{1}, g_{2}$ forms an M-sequence if and only if $g_{2} \leq\left(\begin{array}{c}g_{1}+1 \\ 2\end{array}\right)$. 
The sufficiency of the conditions in Theorem 1.1 was proved by Billera and Lee [BiL], who constructed appropriate polytopes for every M-sequence. Necessity was first proved by Stanley [St1], using the fact that when $P$ is a simplicial polytope, $h_{k}(P)$ is the dimension of the $2 k$ th cohomology group of an associated toric variety $X_{P}$. This variety is projective, and rationally smooth, so the Hard Lefschetz theorem implies that $g_{k}(P)$ is the dimension of the $2 k$ th graded piece of the quotient of $H^{\bullet}\left(X_{P} ; \mathbb{R}\right)$ by the ideal generated by an element $\lambda \in H^{2}\left(X_{P} ; \mathbb{R}\right)$. Since the cohomology ring of a projective toric variety is generated by the elements of degree 2 , so its the quotient by $\lambda$, which shows that the $g$-numbers are an M-sequence.

Note that although $X_{P}$ is only defined when $P$ is rational, by small deformations of the vertices a simplicial polytope can be made rational without changing its combinatorial type, so Stanley's argument established the $g$-theorem for all simplicial polytopes. This deformation trick does not work for general nonsimplicial polytopes, since there are combinatorial types of polytopes which cannot be realized over the rationals; see [Z, Lecture 6.5(a)]. McMullen later gave a proof [McM2] of the necessity part of the $g$-theorem which did not involve toric varieties and so worked for non-rational polytopes without a deformation.

1.2. The toric polynomials. When $P$ is not simplicial, the definition (2) does not behave well - the resulting polynomial can have negative coefficients, and the Dehn-Sommerville relations do not hold. There is a related problem on the topological side - the cohomology of a toric variety defined by a non-simplicial polytope can exist in odd degrees, and the Betti numbers are not invariants of the combinatorics of faces $[\mathrm{McC}]$.

The correct way to generalize the simplicial situation is to replace the cohomology of $X_{P}$ with the intersection (co)homology $I H^{\bullet}\left(X_{P} ; \mathbb{R}\right)$. These groups, defined by Goresky and MacPherson, are just the usual cohomology when $X_{P}$ is rationally smooth, but they are often better behaved than cohomology when the variety is singular. Many important results which hold for cohomology of smooth varieties generalize to intersection cohomology of singular varieties. For instance, the intersection cohomology Betti numbers of the toric variety $X_{P}$ depend only on the face lattice of $P$, even when $P$ is not simplicial.

The dimensions of these groups are given by the the toric $h$-polynomial, which Stanley introduced and analyzed in [St2]. The definition is recursive: $g(P, t)$ is still computed from $h(P, t)$ by the formula (3), but the formula for $h(P, t)$ becomes

$$
h(P, t)=\sum_{F<P} g(F, t)(t-1)^{d-1-\operatorname{dim}(F)},
$$

where the sum is over all faces $F$ of $P$, including the empty face but not $P$ itself. The induction starts by setting $g(P, t)=h(P, t)=1$ when $P$ is the empty 
polytope. It is easy to see that when $P$ is a simplex, $g(P, t)=1$, so this definition agrees with the earlier definition for simplicial polytopes.

The coefficients of these polynomials depend on more than the face numbers of $P$; they are $\mathbb{Z}$-linear combinations of the flag numbers of $P$. When $S \subset$ $\{1, \ldots, d-1\}$, the flag number $f_{S}=f_{S}(P)$ counts the number of chains

$$
F_{i_{1}}<\cdots<F_{i_{r}}
$$

of faces of $P$, where the elements of $S$ are $i_{1}<\cdots<i_{r}$. When $P$ is simplicial, the flag numbers are determined by the face numbers, and so are determined by the $g$-numbers. For general polytopes, however, there is a lot more information in the flag numbers than in the $g$-numbers, although the flag numbers are determined by more general "convolutions" of the $g$-numbers [Kal2].

If $P$ is $d$-dimensional, then formulas for the first three $g$-numbers are $g_{0}(P)=1$ and

$$
\begin{aligned}
& g_{1}(P)=f_{0}-(d+1) \\
& g_{2}(P)=f_{1}+f_{02}-3 f_{2}-d f_{0}+\left(\begin{array}{c}
d+1 \\
2
\end{array}\right) .
\end{aligned}
$$

Formulas for higher $g$-numbers in terms of flag numbers quickly become very complicated, but simpler expressions can be obtained by using a different basis coming from the cd-index [BayE].

Stanley showed [St2] that the toric $h$-numbers satisfy the Dehn-Sommerville equalities: $h_{i}(P)=h_{d-i}(P)$ for all $0 \leq i \leq d$. For rational polytopes, they follow from Poincaré duality for intersection cohomology, but Stanley gave a purely combinatorial proof which works for arbitrary Eulerian posets. While this gives all linear relations among the face numbers of simplicial polytopes, for arbitrary polytopes a much larger set of "generalized Dehn-Sommerville" relations [BayBi] is needed to give all linear relations among flag numbers.

The toric $g$-numbers of $P$ are the dimensions of the graded pieces of the primitive intersection cohomology of $X_{P}$, which is the quotient of $I H \bullet\left(X_{P} ; \mathbb{R}\right)$ by the action of a Lefschetz class in $H^{2}\left(X_{P} ; \mathbb{R}\right)$ (note that intersection cohomology does not have a ring structure, but it is a graded module over the cohomology ring). This implies the nonnegativity of the $g$-numbers when $P$ is rational. The theory of combinatorial intersection cohomology has extended this to arbitrary polytopes.

Another important inequality among the $g$-numbers is monotonicity, which was originally conjectured by Kalai:

Theorem 1.2. for any face $F$ of $P$,

$$
g(P, t) \geq g(F, t) g(P / F, t),
$$


where the inequality is taken coefficient by coefficient.

Here $P / F$ denotes the "quotient polytope" whose face poset is isomorphic to the interval between $F$ and $P$. This was proved for rational polytopes in [BM] using a localization argument on intersection cohomology sheaves. It was pointed out in [BBFK2, BrL1] that this argument translates directly to the combinatorial $\mathrm{IH}$ setting, so the theorem is now known for arbitrary polytopes. We give a partial generalization of this result in Theorem 3.3 below.

The lack of a ring structure on intersection cohomology leaves open the question of whether the toric $g$-numbers give an M-sequence. Besides the fact that it holds for simplicial polytopes, there are two other indications that the answer could be positive. First, we have the generalized upper bound theorem:

Theorem 1.3 ([Bay1]). For any d-polytope $P$ and $1 \leq i \leq d / 2$,

$$
g_{i}(P) \leq\left(\begin{array}{c}
f_{0}-d+i-2 \\
i
\end{array}\right) .
$$

Bayer's proof in [Bay1] relied on Kalai's monotonicity, which at the time was only established for rational polytopes. Now, however, Theorem 1.2 is known for arbitrary polytopes, so Theorem 1.3 is as well. Theorem 1.3 also follows from a result of Stanley [St3, Theorem 7.9(a)] which says that the $g$-numbers can only increase under subdivisions. His proof was valid only for rational polytopes, but it translates immediately to the combinatorial intersection cohomology setting, so again rationality is not necessary.

The second indication the the toric $g$-numbers might be an $M$-sequence is the following unpublished result of Kalai, which is another consequence of Theorem 1.2 .

Theorem 1.4. For a d-polytope $P, g_{k}(P)=0$ implies $g_{k+1}(P)=0$.

We present Kalai's ingenious proof in an appendix.

In general we seem to be far from the goal of characterizing all possible flag $f$-vectors, but many necessary conditions are now known. Since the linear equalities are known [BayBi], the next level of complexity is given by linear inequalities. A summary of the best linear inequalities currently known for dimensions 2 through 8 appears in Ehrenborg [E]. The inequalities $g_{k} \geq 0$ and combinations of them obtained by Kalai's convolution operation [Kal2] provide one main source. Another is the cd-index; we will not discuss this here, but note that Karu [Kar3, Kar2] has recently given a construction similar to the combinatorial intersection cohomology which produces the cd-index. 


\section{COMBINATORIAL INTERSECTION COHOMOLOGY OF FANS}

2.1. Cones and fans. Although we have described the $h$-polynomial as an invariant of polytopes, the combinatorial intersection cohomology is most naturally described in terms of of an associated fan, which carries the same combinatorial structure but has more convenient geometric properties. We first fix some notations and definitions regarding convex cones and fans.

Let $V$ be a finite-dimensional real vector space. A polyhedral cone in $V$ is a subset of the form

$$
\mathbb{R}_{\geq 0} v_{1}+\cdots+\mathbb{R}_{\geq 0} v_{n}, v_{1}, \ldots, v_{n} \in V .
$$

All our cones will be assumed to be pointed, meaning that they do not contain any vector subspace other than $\{0\}$. Any pointed cone $\sigma$ can be expressed as the cone $c P$ over a polytope $P$ of $\operatorname{dimension} \operatorname{dim} P=\operatorname{dim} \sigma-1$, by scaling the vectors $v_{1}, \ldots, v_{n}$ so that they lie in a hyperplane not containing the origin, and letting $P$ be their convex hull. The resulting polytope is well-defined up to projective equivalence. The map $F \mapsto c F$ is an order-preserving bijection between faces of $P$ and faces of $\sigma=c P$, where the empty face $\emptyset$ is considered to be a face of $P$ but not of $\sigma$. Unless we specifically exclude it, we always consider a cone or polytope to be a face of itself.

A fan $\Delta$ in $V$ is a finite collection of cones in $V$ so that every face of a cone in $\Delta$ is again in $\Delta$, and the intersection of any two cones in $\Delta$ is a face of each. We use the notation $\tau \prec \sigma$ to indicate that a cone $\tau$ is a face of $\sigma$. The support $|\Delta|$ of $\Delta$ is the union of all its cones. For instance, the set of all faces of a cone $\sigma$ forms a fan with support $\sigma$; we denote this fan by $[\sigma]$.

A subfan of a fan $\Delta$ is a subset which is itself a fan. For example, given a cone $\sigma$, its boundary $\partial \sigma=[\sigma] \backslash\{\sigma\}$ is a subfan of $[\sigma]$. The relative interior $\sigma^{\circ}$ of $\sigma$ is defined to be $\sigma \backslash|\partial \sigma|$.

A fan $\Delta$ in $V$ is complete if $|\Delta|=V$. A polytope $P$ in $V$ gives rise to a complete fan $\Delta_{P}$ in $V$, known as the central fan of $P$, by choosing the origin to be an interior point of $P$ and coning off all faces $F \neq P$.

Given a fan $\Delta$ and $k \geq 0$, its $k$-skeleton $\Delta_{\leq k}$ is the subfan consisting of all cones of dimension $\leq k$, and we write $\Delta_{k}$ for the set (not a subfan) of all cones in $\Delta$ of dimension exactly $k$.

If $\Delta$ is a fan in $V$ and $\Delta$ is a fan in $V^{\prime}$, then a morphism of fans $\phi: \Delta \rightarrow \Delta^{\prime}$ is a linear map $V \rightarrow V^{\prime}$ so that for every cone $\sigma \in \Delta$ there exists $\tau \in \Delta^{\prime}$ with $\phi(\sigma) \subset \tau$. If $\tau$ can always be chosen so that $\phi$ induces an isomorphism between $\sigma$ and $\tau$, we call $\phi$ a conewise linear isomorphism. One can also define maps between fans which do not come from a global linear map, but we will not need them. 
2.2. Conewise polynomial functions for simplicial fans. For any fan $\Delta$, we say a function $f:|\Delta| \rightarrow \mathbb{R}$ is conewise polynomial if for all $\sigma \in \Delta$ the restriction $\left.f\right|_{\sigma}$ is a polynomial. The set $\mathcal{A}(\Delta)$ of such functions is a graded ring under the operations of pointwise addition and multiplication. Here we use the usual grading where linear functions have degree 1.

Suppose that $\Delta$ is a simplicial fan, meaning that each cone is a cone over a simplex. As an abstract poset, $\Delta$ is isomorphic to a simplicial complex, and the ring $\mathcal{A}(\Delta)$ is isomorphic to the face ring of this complex, also known as the Stanley-Reisner ring. This is a quotient of the polynomial ring $\mathbb{R}\left[x_{\rho}\right]$ with one generator for each 1-cone $\rho \in \Delta_{1}$ by the ideal

$$
\left.\left\langle x_{\rho_{1}} \cdots x_{\rho_{k}}\right| \rho_{1}, \ldots, \rho_{k} \text { are not the } 1 \text {-faces of a cone in } \Delta\right\rangle \text {. }
$$

The isomorphism comes by identifying the generator $x_{\rho}$ with a conewise linear function which restricts to a nonzero function on $\rho$ and to zero on all other $\rho^{\prime} \in \Delta_{1}$.

A simple inclusion-exclusion argument on monomials in the $x_{\rho}$ shows that the Hilbert series of $\mathcal{A}(\Delta)$ is

$$
\operatorname{Hilb}(\mathcal{A}(\Delta), t)=h(\Delta, t) /(1-t)^{\operatorname{dim} V},
$$

where $h(\Delta, t)=\sum_{i}\left|\Delta_{i}\right|(t-1)^{\operatorname{dim} V-i}$ is the corresponding $h$-polynomial. When $\Delta=\Delta_{P}$ is the central fan of a simplicial polytope $P$, then $h(\Delta, t)=h(P, t)$.

Realizing the face ring of a simplicial complex as the ring of conewise polynomial functions on a fan gives an extra structure to the face ring in the following way. Let $A=\operatorname{Sym}\left(V^{*}\right)$ be the ring of polynomial functions on $V$. There is a natural ring homomorphism $A \rightarrow \mathcal{A}(\Delta)$ obtained by restricting polynomials to $|\Delta|$; it is injective if $\Delta_{\operatorname{dim} V} \neq \emptyset$. This makes $\mathcal{A}(\Delta)$ into an algebra over $A$, and in particular a graded $A$-module.

For any graded $A$-module $M$ we define $\bar{M}=M / \mathfrak{m} M=M \otimes_{A} A / \mathfrak{m}$, the quotient by the maximal ideal generated by $A_{1}$. Now assume that $\Delta$ is a $d$-dimensional complete simplicial fan. Then one can show that $\mathcal{A}(\Delta)$ is a free $A$-module. Since the Hilbert series of $A$ is $(1-t)^{-\operatorname{dim} V}$, this gives

$$
\operatorname{Hilb}(\overline{\mathcal{A}(\Delta)}, t)=h(\Delta, t) .
$$

This shows that the $h$-numbers of a complete fan $\Delta$ are nonnegative; since $h\left(\Delta_{P}, t\right)=h(P, t)$, this gives the nonnegativity of the $h$-numbers of a polytope $P$. By the Dehn-Sommerville relations, we have

$$
\operatorname{dim} \overline{\mathcal{A}(\Delta)}_{k}=\operatorname{dim} \overline{\mathcal{A}(\Delta)}_{d-k}, 0 \leq k \leq d .
$$

Brion [Bri] showed that this can be lifted to an $A$-linear dual pairing

$$
\mathcal{A}(\Delta) \otimes_{A} \mathcal{A}(\Delta) \rightarrow A[d],
$$


where $[d]$ shifts the degree down by $d$. The pairing is well-defined up to multiplication by a positive scalar.

The nonnegativity of the $g$-numbers follows from the existence of a Lefschetz element for $\mathcal{A}(\Delta)$, which is an element $\ell \in \mathcal{A}(\Delta)_{1}$ for which the multiplication

$$
\ell^{d-2 k}: \overline{\mathcal{A}(\Delta)}_{k} \rightarrow \overline{\mathcal{A}(\Delta)}_{d-k}
$$

is an isomorphism for $0 \leq k<d / 2$. For such an $\ell$, multiplication $\ell \cdot: \overline{\mathcal{A}(\Delta)}_{k} \rightarrow$ $\overline{\mathcal{A}(\Delta)}_{k+1}$ is an injection for $k<d / 2$ and a surjection for $k>d / 2-1$, and it follows that the quotient ring $H=\overline{\mathcal{A}(\Delta)} / \ell \overline{\mathcal{A}(\Delta)}$ has Hilbert series $g(\Delta, t)$. Thus the existence of a Lefschetz element implies that the $g$-numbers are nonnegative. It also shows that they form an M-sequence, since $H$ is generated by elements of degree 1 .

When $P$ is a rational simplicial polytope the $\operatorname{ring} \overline{\mathcal{A}(\Delta)}$ is canonically isomorphic to the cohomology ring $H^{\bullet}\left(X_{P} ; \mathbb{R}\right)$, by a map which doubles degree. The variety $X_{P}$ is projective, and an embedding into a projective space determines an ample class in $H^{2}\left(X_{P} ; \mathbb{R}\right.$ ). The Hard Lefschetz theorem (or more precisely its extension to rationally smooth varieties due to Saito and Beilinson-BernsteinDeligne) implies that this class is a Lefschetz element. This was essentially Stanley's argument to prove the necessity part of the $g$-theorem.

McMullen later gave a more elementary proof without using algebraic geometry. A conewise linear function $\ell$ is called "strictly convex" if it is convex and it gives a different linear function on each full-dimensional cone. Such a function exists if and only if $\Delta$ is polytopal, meaning that it is the central fan $\Delta_{P}$ of a convex polytope $P$. This is because the graph of a strictly convex function is the boundary of a cone isomorphic to the cone $c P$.

Theorem 2.1 (McMullen [McM2]). If $\Delta$ is a complete simplicial fan, then any strictly convex function $\ell \in \mathcal{A}(\Delta)_{1}$ is a Lefschetz element for $\mathcal{A}(\Delta)$.

Strictly convex linear functions correspond exactly to ample classes in $H^{2}\left(X_{P} ; \mathbb{R}\right)$ when $P$ is rational, so McMullen's result gives an elementary proof of the Hard Lefschetz theorem for rationally smooth toric varieties.

McMullen actually proved the stronger Hodge-Riemann inequalities, which say that under the Poincaré pairing between $\overline{\mathcal{A}(\Delta)}_{k}$ and $\overline{\mathcal{A}(\Delta)}_{d-k}$ the map $(-1)^{k} \ell^{d-2 k}$ is positive definite on the kernel of $\ell^{d-2 k+1}$. His proof used induction on dimension, and these inequalities were essential to make the induction work. McMullen's original argument used a different algebraic structure, the polytope algebra, in place of the ring $\overline{\mathcal{A}(\Delta)}$; in this language the Hodge-Riemann inequalities become beautiful statements about mixed volumes and Minkowski geometry. The relation between the polytope algebra and cohomology of toric varieties is described in [Bri, FuS]. Timorin [Ti] later gave a nice presentation of McMullen's 
argument using another description of the ring $\overline{\mathcal{A}(\Delta)}$ due to Khovanskii and Pukhlikov.

Remark. For the Hodge-Riemann inequalities to hold, it is essential that $\ell$ be strictly convex. The condition that $\ell$ be a Lefschetz element is considerably weaker, however. In fact, the set of Lefschetz elements is a Zariski open subset of $\mathcal{A}(\Delta)_{1}$, so if it is nonempty, then it is dense. This suggests that it might be possible to find Lefschetz elements under weaker assumptions than strict convexity. This would be useful to show that the conditions of the $g$-theorem hold for more general simplicial spheres.

2.3. Example. Let $\Delta$ be the fan in $\mathbb{R}^{2}$ whose maximal cones are the four quadrants $\{(x, y) \mid \pm x \geq 0, \pm y \geq 0\}$. Then $h(\Delta, t)=1+2 t+t^{2}$, and the functions

$$
1,|x|,|y|,|x y|
$$

give an $A$-module basis for $\mathcal{A}(\Delta)$. To see this, it is easier to show that

$$
1,|x|-x,|y|-y,(|x|-x)(|y|-y)
$$

is a basis - one can subtract off multiples of these to cancel any element, one quadrant at a time, and this can be done uniquely.

The strictly convex function $\ell=|x|+|y|$ gives a Lefschetz element; in this case this just means that $\ell^{2}=x^{2}+y^{2}+2|x y|$ gives a nonzero element in $\overline{\mathcal{A}(\Delta)_{2}}$.

2.4. Sheaves on fans. For a general fan $\Delta$, the $\operatorname{ring} \mathcal{A}(\Delta)$ is not as well-behaved as it is for simplicial fans. For instance, it is not a free $A$-module, and $\overline{\mathcal{A}(\Delta)}{ }_{k}$ and $\overline{\mathcal{A}(\Delta)}_{d-k}$ may not have the same dimension. The correct generalization from the simplicial case is the theory of combinatorial intersection cohomology of [BBFK2, BrL1]. It is expressed in terms of sheaves on the fan $\Delta$, and we begin with a brief review of this formalism.

We consider a fan $\Delta$ as a topological space by taking as open subsets all of its subfans. Given an arbitrary subset $\Sigma \subset \Delta$, there is a unique smallest subfan/open set of $\Delta$ containing $\Sigma$, namely $[\Sigma]=\bigcup_{\sigma \in \Sigma}[\sigma]$. The closure of a single cone $\{\sigma\}$ in this topology is the star

$$
\operatorname{st}(\sigma)=\operatorname{st}_{\Delta}(\sigma)=\{\tau \in \Delta \mid \sigma \prec \tau\} .
$$

A sheaf $\mathcal{F}$ of vector spaces on $\Delta$ is given by an assignment $\Sigma \mapsto \mathcal{F}(\Sigma)$ of a vector space to each subfan $\Sigma \subset \Delta$, together with restriction maps $\mathcal{F}(\Sigma) \rightarrow \mathcal{F}\left(\Sigma^{\prime}\right)$ for any pair of subfans $\Sigma^{\prime} \subset \Sigma$. These are required to satisfy (1) if $\Sigma^{\prime \prime} \subset \Sigma^{\prime} \subset \Sigma$ are subfans of $\Delta$, then the obvious triangle of restrictions is commutative, and (2) if $\Sigma, \Sigma^{\prime}$ are any two subfans of $\Delta$, then the image of $\mathcal{F}\left(\Sigma \cup \Sigma^{\prime}\right)$ in $\mathcal{F}(\Sigma) \oplus \mathcal{F}\left(\Sigma^{\prime}\right)$ is the set of pairs $(x, y)$ for which $x$ and $y$ restrict to the same element of $\mathcal{F}\left(\Sigma \cap \Sigma^{\prime}\right)$. Sheaves of rings or modules are defined in the same way. 
For any fan $\Delta$, the assignment $\Sigma \mapsto \mathcal{A}(\Sigma)$ gives a sheaf known as the sheaf of conewise polynomial functions. We denote it by $\mathcal{A}_{\Delta}$ or simply $\mathcal{A}$ if the fan is understood. It is a sheaf of rings, which means we can define sheaves of modules over it, as follows. An $\mathcal{A}_{\Delta}$-module is a sheaf $\mathcal{F}$ on $\Delta$ together with a structure of a graded $\mathcal{A}(\Sigma)$-module on $\mathcal{F}(\Sigma)$ for every subfan $\Sigma \subset \Delta$. This is required to be compatible with the restriction maps: $\mathcal{F}\left(\Sigma^{\prime}\right) \rightarrow \mathcal{F}(\Sigma)$ should be a homomorphism of $\mathcal{A}\left(\Sigma^{\prime}\right)$-modules whenever $\Sigma \subset \Sigma^{\prime}$ are subfans of $\Delta$, where $\mathcal{F}(\Sigma)$ becomes a $\mathcal{A}\left(\Sigma^{\prime}\right)$-module via the restriction $\mathcal{A}\left(\Sigma^{\prime}\right) \rightarrow \mathcal{A}(\Sigma)$.

Because $\Delta$ is finite, a sheaf $\mathcal{F}$ on $\Delta$ can be described by a finite collection of modules and maps. The stalk of $\mathcal{F}$ at a cone $\sigma \in \Delta$ is $\mathcal{F}([\sigma])$, since $[\sigma]$ is the smallest open set containing $\sigma$. To simplify notation, we write $\mathcal{F}(\sigma)$ for $\mathcal{F}([\sigma])$. If $\tau \prec \sigma$, then restriction of sections gives a homomorphism $\mathcal{F}(\sigma) \rightarrow \mathcal{F}(\tau)$. Since any subfan of $\Delta$ is a union of fans of the form $[\sigma]$, the data in the sheaf $\mathcal{F}$ is equivalent to the collection of stalks together with the restriction maps between them (subject to the obvious commutation relation).

The stalks of $\mathcal{A}_{\Delta}$ are particularly simple: $\mathcal{A}_{\Delta}(\sigma)$ is the ring of polynomial functions on the span of $\sigma$, which we denote by $A_{\sigma}$. If $\mathcal{F}$ is an $\mathcal{A}_{\Delta}$-module, then the $\mathcal{F}(\sigma)$ is a $A_{\sigma}$-module for every face $\sigma$.

For a $\Delta$-sheaf $\mathcal{F}$ and a pair of subfans $\Sigma \subset \Sigma^{\prime}$ of $\Delta$ we define the space of relative sections $\mathcal{F}\left(\Sigma^{\prime}, \Sigma\right)$ to be the kernel of the restriction $\mathcal{F}\left(\Sigma^{\prime}\right) \rightarrow \mathcal{F}(\Sigma)$.

If $\phi: \Delta \rightarrow \Delta^{\prime}$ is a morphism of fans, and $\mathcal{F}$ is a sheaf on $\Delta$, then the pushforward sheaf $\phi_{*} \mathcal{F}$ on $\Delta^{\prime}$ is defined by $\phi_{*} \mathcal{F}(\Sigma)=\mathcal{F}\left(\phi^{-1}(\Sigma)\right)$, where

$$
\phi^{-1}(\Sigma)=\{\tau \in \Delta|\phi(\tau) \subset| \Sigma \mid\} .
$$

If $\phi$ is a conewise linear isomorphism, then $\phi_{*} \mathcal{A}_{\Delta} \cong \mathcal{A}_{\Delta^{\prime}}$; in general we only have a natural map $\mathcal{A}_{\Delta^{\prime}} \rightarrow \phi_{*} \mathcal{A}_{\Delta}$. If $\mathcal{F}$ is an $\mathcal{A}_{\Delta}$-module, then this map makes $\phi_{*} \mathcal{F}$ into an $\mathcal{A}_{\Delta^{\prime}}$-module.

2.5. Combinatorial IH sheaves. The main definition in the theory of combinatorial intersection cohomology developed in [BBFK2, BrL1] is the following.

Definition 2.2. An $\mathcal{A}_{\Delta}$-module $\mathcal{F}$ is called pure if it is

- locally free, meaning that $\mathcal{F}(\sigma)$ is a free $A_{\sigma}$-module for every $\sigma \in \Delta$, and

- flabby, meaning that the restriction $\mathcal{F}(\Delta) \rightarrow \mathcal{F}(\Sigma)$ is surjective for any subfan $\Sigma$ of $\Delta$.

Note that flabbiness of $\mathcal{F}$ can be expressed in two other equivalent ways:

(1) $\mathcal{F}(\Sigma) \rightarrow \mathcal{F}\left(\Sigma^{\prime}\right)$ is surjective for any subfans $\Sigma^{\prime} \subset \Sigma$ of $\Delta$, or

(2) $\mathcal{F}(\sigma) \rightarrow \mathcal{F}(\partial \sigma)$ is surjective for every $\sigma \in \Delta$. 
Indecomposable pure sheaves are constructed inductively as follows. Starting with a cone $\sigma \in \Delta$, define a sheaf $\mathcal{L}={ }_{\sigma} \mathcal{L}_{\Delta}$ to be zero on all cones $\tau \notin \operatorname{st}(\sigma)$, and let $\mathcal{L}(\sigma)=A_{\sigma}$. Then, assuming that $\mathcal{L}$ has already been defined on $\partial \tau$ for $\tau \in \Delta$, let $\mathcal{L}(\tau)$ be a minimal free $A_{\tau}$-module which surjects onto $\mathcal{L}(\partial \tau)$, where $\mathcal{L}(\partial \tau)$ is an $A_{\tau}$-module via the restriction $A_{\tau}=\mathcal{A}(\tau) \rightarrow \mathcal{A}(\partial \tau)$. Equivalently, let $\mathcal{L}(\tau)$ be a free $A_{\tau}$-module generated by a vector space basis for $\overline{\mathcal{L}(\partial \tau)}$, and let the map $\mathcal{L}(\tau) \rightarrow \mathcal{L}(\partial \tau)$ be given by choosing representatives for the basis elements.

The following "decomposition theorem" is an easy consequence of these definitions.

Theorem 2.3 ([BBFK2, BrL1]). The sheaves ${ }_{\sigma} \mathcal{L}_{\Delta}, \sigma \in \Delta$ give a complete list of isomorphism classes of indecomposable pure $\mathcal{A}_{\Delta}$-modules, up to shifts of degree. Every pure sheaf is isomorphic to a direct sum of indecomposable objects.

The sheaves ${ }_{\sigma} \mathcal{L}_{\Delta}$ are the combinatorial IH sheaves. For many questions it is enough to look at ${ }_{o} \mathcal{L}_{\Delta}$, where $o=\{0\}$ is the zero cone, since the other combinatorial IH sheaves can be derived from it by a pullback and base change from a quotient fan. To simplify notation, we put $\mathcal{L}_{\Delta}={ }_{o} \mathcal{L}_{\Delta}$. Note that if $\Sigma \subset \Delta$ is a subfan containing the cone $\sigma$, then the restriction of ${ }_{\sigma} \mathcal{L}_{\Delta}$ to $\Sigma$ is ${ }_{\sigma} \mathcal{L}_{\Sigma}$, so we can write $\sigma \mathcal{L}(\Sigma)$ for the sections on $\Sigma$ without causing confusion.

If $\Delta$ is simplicial, then $\mathcal{A}_{\Delta}$ is pure, so $\mathcal{L}_{\Delta}$ is (canonically) isomorphic to $\mathcal{A}_{\Delta}$. Thus global sections of $\mathcal{L}$ are just conewise polynomial functions; if $\Delta=\Delta_{P}$ for a simplicial polytope $P$, then $\operatorname{Hilb}(\overline{\mathcal{L}(\Delta)}, t)$ is the simplicial $h$-polynomial defined by $(2)$.

This generalizes to arbitrary polytopes, using the toric $g$ and $h$-numbers:

Theorem 2.4. Let $P$ be a polytope.

(a) If $\Delta=\Delta_{P}$ is the central fan of a polytope $P$, and $\sigma=c F$ is the cone over a proper face $F$ of $P$, then ${ }_{\sigma} \mathcal{L}(\Delta)$ is a free $A$-module and

$$
\operatorname{Hilb}(\overline{\sigma \mathcal{L}(\Delta)}, t)=h(P / F, t) .
$$

(b) If $\tau=c P$ and $\sigma=c F$ for a face $F \leq P$, then $\operatorname{Hilb}(\overline{\sigma \mathcal{L}(\tau)}, t)=g(P / F, t)$.

Unlike the simplicial case, the proof of this is far from straightforward. In order to explain the basic structure of the proof, we restrict for simplicity to the case $\sigma=o$. Let $(\mathrm{a})_{d}$ and (b) ${ }_{d}$ represent the statements (a) and (b), restricted to polytopes $P$ of dimension $\leq d$. Theorem 2.4 is proved by a spiraling induction, by showing that $(\mathrm{b})_{d-1} \Longrightarrow(\mathrm{a})_{d}$ and $(\mathrm{a})_{d} \Longrightarrow(\mathrm{b})_{d}$.

The first implication follows from the statement that for a complete fan the cellular cohomology gives an exact sequence

$$
0 \rightarrow \mathcal{L}(\Delta) \rightarrow \bigoplus_{\sigma \in \Delta_{d}} \mathcal{L}(\sigma) \rightarrow \bigoplus_{\tau \in \Delta_{d-1}} \mathcal{L}(\tau) \rightarrow \cdots \rightarrow \mathcal{L}(o) \rightarrow 0
$$


where the maps are sums of all possible restriction maps, with appropriate minus signs added to make it a complex. Together with some simple commutative algebra this implies that $\mathcal{L}(\Delta)$ is a free $A$-module. Taking Hilbert series, we obtain

$$
(1-t)^{-d} \operatorname{Hilb}\left(\overline{\mathcal{L}\left(\Delta_{P}\right)}, t\right)=\sum_{\sigma \in \Delta}(-1)^{d-\operatorname{dim} \sigma} \operatorname{Hilb}(\overline{\mathcal{L}(\sigma)}, t)(1-t)^{-\operatorname{dim} \sigma},
$$

which shows that $\operatorname{Hilb}\left(\overline{\mathcal{L}\left(\Delta_{P}\right)}, t\right)=h(P, t)$, using (4) and the assumption $(\mathrm{b})_{d-1}$.

The other implication $(\mathrm{a})_{d} \Longrightarrow(\mathrm{b})_{d}$ is more subtle. Take $P$ a $d$-polytope, and let $\sigma=c P$. Let $V$ be the linear span of $\sigma$. If $v$ is in the relative interior of $\sigma$, then the projection $\pi: V \rightarrow W=V / \mathbb{R} v$ gives a conewise linear isomorphism from $\partial \sigma$ to a complete fan $\Delta$ in $W$ which is isomorphic to the central fan $\Delta_{P}$. Conversely, $\partial \sigma$ can be viewed as the graph of a strictly convex conewise linear function $\ell \in \mathcal{A}(\Delta)_{1}$.

Since $\pi$ induces a linear isomorphism between cones of $\partial \sigma$ and cones of $\Delta$, there is an isomorphism of $\mathcal{A}_{\Delta}$-sheaves $\pi_{*} \mathcal{L}_{\partial \sigma} \cong \mathcal{L}_{\Delta}$. Taking global sections, we get an isomorphism

$$
\mathcal{L}(\partial \sigma) \cong \mathcal{L}(\Delta)
$$

of $A^{\prime}$-modules, where $A^{\prime}=\operatorname{Sym}\left(W^{*}\right)$ is included as a subring of $A=\operatorname{Sym}\left(V^{*}\right)$ by the pullback $\pi^{*}$. Choosing a degree 1 element $y \in A \backslash A^{\prime}$ gives an isomorphism $A \cong A^{\prime}[y]$. In terms of the isomorphism $(7)$, the action of $y$ on $\mathcal{L}(\Delta)$ is given by multiplication by $\ell$.

By the construction of $\mathcal{L}$, the map $\overline{\mathcal{L}(\sigma)} \rightarrow \overline{\mathcal{L}(\partial \sigma)}$ is an isomorphism. On the other hand, the isomorphism (7) implies that $\overline{\mathcal{L}(\partial \sigma)} \cong \overline{\mathcal{L}(\Delta)} / \ell \overline{\mathcal{L}(\Delta)}$. The inductive hypothesis $(\mathrm{a})_{d}$ says that the Hilbert series of $\overline{\mathcal{L}(\Delta)}$ is $h(P, t)$. Thus (b) ${ }_{d}$ follows from the following difficult result of Karu.

Theorem 2.5 ([Kar1]). If $\Delta=\Delta_{P}$ for a polytope $P$, then any strictly convex conewise linear function $\ell$ acts on $\overline{\mathcal{L}(\Delta)}$ as a Lefschetz operator.

As with McMullen's Theorem 2.1, what Karu actually proved is the stronger Hodge-Riemann inequalities, and they are necessary to make the induction work. The inequalities are taken with respect to a dual pairing

$$
\overline{\mathcal{L}(\Delta)}_{k} \otimes \overline{\mathcal{L}(\Delta)}_{d-k} \rightarrow \mathbb{R}
$$

Karu used a pairing from [BBFK2] which involved choices, but Bressler and Lunts [BrL2] have simplified Karu's argument by using a canonical pairing they defined in [BrL1]. Another presentation of Karu's theorem appears in [BBFK4], using another approach to defining a canonical pairing from [BBFK3].

Karu's theorem implies the following degree vanishing result. 
Theorem 2.6. For $\sigma \prec \tau$, the stalk ${ }_{\sigma} \mathcal{L}(\tau)$ is generated in degrees $<(\operatorname{dim} \tau-$ $\operatorname{dim} \sigma) / 2$, while the costalk

$$
{ }_{\sigma} \mathcal{L}(\tau, \partial \tau)=\operatorname{ker}\left({ }_{\sigma} \mathcal{L}(\tau) \rightarrow{ }_{\sigma} \mathcal{L}(\partial \tau)\right)
$$

is generated in degrees $>(\operatorname{dim} \tau-\operatorname{dim} \sigma) / 2$.

In particular, ${ }_{\sigma} \mathcal{L}(\tau) \rightarrow{ }_{\sigma} \mathcal{L}(\partial \tau)$ is an isomorphism in degrees $<(\operatorname{dim} \tau-$ $\operatorname{dim} \sigma) / 2$. This has the following important consequence.

Corollary 2.7. The only automorphisms of the combinatorial IH sheaf $\sigma \mathcal{L}$ (as a graded $\mathcal{A}_{\Delta}$-module) are multiplication by scalars $\mathbb{R}^{\times}$.

This means that the combinatorial IH groups are canonically associated to the fan. As a result, the groups themselves carry interesting information about $P$ beyond just their dimensions.

2.6. Connections with topology. Although the theory of combinatorial IH sheaves can be developed without referring to toric varieties, the topological interpretation still provides a powerful way of understanding what these results mean. All the cohomology and intersection cohomology spaces below are taken with $\mathbb{R}$ coefficients.

Suppose that the fan $\Delta$ is rational with respect to a fixed lattice in the vector space $V$. The definition of the combinatorial IH sheaf $\mathcal{L}_{\Delta}$ was first given by Barthel, Brasselet, Fieseler and Kaup in [BBFK1], where they show that the $\mathcal{L}(\Delta)$ is isomorphic to the equivariant intersection cohomology $I H_{T}^{\bullet}\left(X_{\Delta}\right)$ of the associated toric variety under the action of the usual torus $T \cong\left(\mathbb{C}^{*}\right)^{d}$.

Equivariant intersection cohomology, which was defined in [BeL, Bry, J], is a topological invariant associated to a space endowed with an action of a group. When the group is trivial, it specializes to the usual intersection cohomology, and when the space is smooth, it gives the equivariant cohomology. The extra information in the group action gives the equivariant cohomology and intersection cohomology more structure and better properties than their non-equivariant counterparts. In particular, they are modules over the equivariant cohomology of a point $H_{T}^{\bullet}(p)$, which is canonically isomorphic to $A=\operatorname{Sym}\left(V^{*}\right)$ by an isomorphism which identifies $V^{*}$ with $H_{T}^{2}(p)$ (note that our choice to use the standard grading on $A$ means that all our isomorphisms between the algebraic and topological sides will double degree).

If $\Sigma \subset \Delta$ is a subfan, then $X_{\Sigma}$ is an open $T$-invariant subvariety of $X_{\Delta}$. Equivariant IH restricts via open inclusions, so there is a homomorphism $I H_{T}^{\bullet}\left(X_{\Delta}\right) \rightarrow$ $I H_{T}^{\bullet}\left(X_{\Sigma}\right)$ of $A$-modules. Barthel, Brasselet, Fieseler and Kaup showed that with these restrictions, the assignment

$$
\Sigma \mapsto I H_{T}^{\bullet}\left(X_{\Sigma}\right), \Sigma \subset \Delta
$$


is a sheaf on $\Delta$.

The fact that this is a presheaf is purely formal - the same is true for the ordinary cohomology and intersection cohomology, for instance. But being a sheaf is special to equivariant intersection cohomology; for most types of cohomology it is not possible to describe classes locally by gluing them from classes on sets in an open cover.

The equivariant cohomology also restricts along open inclusions, but it does not give a sheaf in general (see [BBFK1] for an example). However, it has a sheafification, which is just the sheaf $\mathcal{A}_{\Delta}$ of conewise polynomial functions. In particular, if $\tau$ is a cone, then $H_{T}^{\bullet}\left(X_{[\tau]}\right) \cong A_{\tau}$ canonically. Equivariant intersection cohomology is always a module over equivariant cohomology, which makes the sheaf (8) into an $\mathcal{A}_{\Delta}$-module.

To show that this sheaf is the combinatorial IH sheaf $\mathcal{L}_{\Delta}$, one needs to show that $I H_{T}^{\bullet}\left(X_{[\tau]}\right)$ is the minimal free $A_{\tau}$-module which surjects onto $I H_{T}^{\bullet}\left(X_{\partial \tau}\right)$ for every cone $\tau \neq o$. This was shown in [BBFK1]. It also follows from the following result of Bernstein and Lunts.

Theorem 2.8. [BeL] Let $X$ be a variety with a T-action, and suppose that there is a homomorphism $\rho: \mathbb{C}^{*} \rightarrow T$ which contracts $X$ to a point $y$ :

$$
\lim _{t \rightarrow 0} \rho(t) \cdot x=y \text { for all } x \in X .
$$

Then under restriction $I H_{T}^{\bullet}(X)$ is the smallest free $H_{T}^{\bullet}(p)$-module which surjects onto $I H_{T}^{\bullet}(X \backslash\{p\})$.

The proof in $[\mathrm{BeL}]$ used the Hard Lefschetz theorem for intersection cohomology. A different proof, using the weight filtration on equivariant intersection cohomology, appears in [BM2].

When $\Delta$ is a complete fan or $\Delta=[\tau]$, the variety $X_{\Delta}$ is "formal" for equivariant intersection cohomology in the sense of [GKM]. This means that $I H_{T}^{\bullet}\left(X_{\Delta}\right)$ is a free $A$-module and

$$
I H^{\bullet}\left(X_{\Delta}\right)=\overline{I H_{T}^{\bullet}\left(X_{\Delta}\right)}
$$

canonically. Barthel, Brasselet, Fieseler and Kaup [BBFK2] gave a complete characterization of when $X_{\Delta}$ is formal; we will use it in Section 3.1 below.

When $X_{\Delta}$ is formal, then $\overline{\mathcal{L}(\Delta)}$ is canonically isomorphic to $I H \bullet\left(X_{\Delta}\right)$. So Karu's Theorem 2.5 is not simply a substitute for the Hard Lefschetz theorem; it is actually an alternate proof of it, valid for toric varieties. A careful study of Karu's proof and the combinatorial IH sheaves in general should give new insight into the Hard Lefschetz theorem, which is one of the deepest results in complex algebraic geometry. 
This will hopefully lead to similar elementary proofs in other interesting contexts. For instance, [BM2] defines a similar theory of combinatorial IH sheaves for Schubert varieties; the role of fans is played by "moment graphs," which are linearly embedded graphs encoding the fixed points and invariant curves for a torus action. At present these results are only valid when the graph actually comes from a variety, as there is no result comparable to Karu's theorem so far. In fact, we don't even have an elementary proof of Hard Lefschetz for "smooth" moment graphs, which have been extensively studied by Guillemin and Zara [GZ1, GZ2, GZ3, GZ4].

There is a natural class of non-rational moment graphs for which the construction of [BM2] should give good answers: the "Bruhat graphs" arising from a non-crystallographic Coxeter group $W$. These can be thought of as the moment graphs of the (nonexistent) Schubert varieties in the flag variety of the (nonexistent) semi-simple group with Weyl group $W$, just as non-rational fans correspond to nonexistent toric varieties. The stalks of the combinatorial $\mathrm{IH}$ sheaf on a Bruhat graph for $W$ should be free, with ranks given by KazhdanLusztig polynomials for $W$; this would give a proof that these polynomials have nonnegative coefficients. See [Fieb] for an exposition of these ideas.

Not only does the space of sections of the sheaf $\mathcal{L}$ give the module $I H_{T}^{\bullet}\left(X_{\Delta}\right)$ when $\Delta$ is rational, but the sheaf $\mathcal{L}_{\Delta}$ itself can be seen as a model for the equivariant intersection cohomology sheaf $I C \cdot\left(X_{\Delta}\right)$, an object in the equivariant derived category $D_{T}^{b}\left(X_{\Delta}\right)$ [BeL], and complexes of pure sheaves are models of more general objects of $D_{T}^{b}\left(X_{\Delta}\right)$. This will be discussed in more detail in section 4 .

2.7. Examples in low degree: degree zero and one. To illustrate the features of this theory, we will describe the graded pieces of the sheaf $\mathcal{L}={ }_{o} \mathcal{L}_{\Delta}$ in degrees $\leq 2$. The answers are given as cohomology of simple chain complexes whose terms have dimensions corresponding directly to terms in the formulas (5) and (6) for $g_{1}$ and $g_{2}$.

The degree zero part of $\mathcal{L}$ is trivial; we have

$$
\mathcal{L}(\Delta)_{0}=\mathbb{R}
$$

for any fan $\Delta$.

In order to understand the degree $k$ part of $\mathcal{L}$ for $k \geq 1$, it is enough to understand its restriction to the $(2 k-1)$-skeleton $\Delta_{\leq 2 k-1} \subset \Delta$. This is because Theorem 2.6 implies that the restriction

$$
\mathcal{L}(\Delta)_{k} \rightarrow \mathcal{L}\left(\Delta_{\leq 2 k-1}\right)_{k}
$$

is an isomorphism. 
For $k=1$, this means that it is enough to consider sections of $\mathcal{L}$ on $\Delta_{\leq 1}$. Since this is a simplicial fan, we have

$$
\mathcal{L}(\Delta)_{1}=\mathcal{L}\left(\Delta_{\leq 1}\right)_{1}=\mathcal{A}\left(\Delta_{\leq 1}\right)_{1}=\bigoplus_{\rho \in \Delta_{1}}\langle\rho\rangle,
$$

where we introduce the notation $\langle\tau\rangle=(\operatorname{span} \tau)^{*}$ for the space of linear functions on a cone $\tau$.

Reducing modulo $\mathfrak{m}$, we see that $\overline{\mathcal{L}(\Delta)}$, is the cokernel of the multiplication map $\mu_{1}: V^{*} \otimes \mathcal{L}(\Delta)_{0} \rightarrow \mathcal{L}(\Delta)_{1}$. In terms of the identifications (9) and (10), this is the map

$$
V^{*} \rightarrow \bigoplus_{\rho \in \Delta_{1}}\langle\rho\rangle
$$

induced from the natural restrictions.

If the 1-cones $\rho \in \Delta_{1}$ span $V$, for instance if $\Delta$ contains a full-dimensional cone, then $\mu_{1}$ is injective, so $\operatorname{dim} \overline{\mathcal{L}(\Delta)}{ }_{1}=\# \Delta_{1}-\operatorname{dim}(V)$. Thus for a $d$-polytope $P$ we have

$$
\begin{aligned}
\operatorname{dim}{\overline{\mathcal{L}\left(\Delta_{P}\right)_{1}}}_{1} & =f_{0}(P)-d=h_{1}(P) \\
\operatorname{dim} \overline{\mathcal{L}(c P)_{1}} & =f_{0}(P)-(d+1)=g_{1}(P),
\end{aligned}
$$

as predicted by Theorem 2.4 .

This implies that $g_{1}$ and $h_{1}$ are nonnegative, which is just the obvious statement that a $d$-polytope must have at least $d+1$ vertices. Still, it is interesting to have canonical geometrically defined vector spaces of dimensions $g_{1}(P)$ and $h_{1}(P)$. For instance, the dual of $\overline{\mathcal{L}(c P)}$ is canonically the space of all affine dependencies among the vertices of $P$. This interpretation of $g_{1}$ was used by Kalai to prove a number of results, including low-degree cases of his monotonicity conjecture.

The fact that nothing new needs to be added in degree one for cones of dimension $>1$ can also be seen directly without using Theorem 2.6. What is needed is to see that for any cone $\tau$, the $A_{\tau}$-module defined by (9) and (10) with module structure given by (11) is isomorphic to the degree zero and one part of a free module. But this is just the obvious fact that

$$
\langle\tau\rangle \rightarrow \bigoplus_{\rho \in[\tau]_{1}}\langle\rho\rangle
$$

is injective for all cones $\tau$.

2.8. Degree two. To understand $\mathcal{L}$ in degree two it is enough to understand its restriction to the 3 -skeleton $\Delta_{\leq 3}$. Since any two-cone is simplicial, $\mathcal{L}$ will agree with $\mathcal{A}$ on $\Delta_{\leq 2}$. There will be a correction for nonsimplicial 3-cones, however, since $\mathcal{A}$ will not be flabby on such cones. 
To describe this correction, fix a choice of a subdivision $\widetilde{\Delta}$ of $\Delta_{\leq 3}$ which divides each 3-cone into simplicial cones without adding new 1-cones; in particular the 2-cones are not subdivided. Let $\phi: \widetilde{\Delta} \rightarrow \Delta$ be the associated map of fans. Then the pushforward $\phi_{*} \mathcal{A}_{\widetilde{\Delta}}$ is isomorphic to the restriction of $\mathcal{L}$ to $\Delta_{\leq 3}$. The restrictions of these sheaves to the two-skeleton $\Delta_{\leq 2}$ are clearly isomorphic, since $\mathcal{A}_{\Delta_{\leq 2}}=\mathcal{L}_{\Delta_{\leq 2}}$ because $\Delta_{\leq 2}$ is simplicial. Constructing the isomorphism on a 3 cone $\sigma$ amounts to showing that $\mathcal{A}(\widetilde{[\sigma]})$ is a free $A_{\sigma}$-module generated in degrees zero and one, and that the restriction $\mathcal{A}(\widetilde{[\sigma]}) \rightarrow \mathcal{A}(\partial \sigma)$ is surjective, with kernel generated in degrees $\geq 2$.

By Corollary 2.7, this isomorphism is canonical. As a consequence, the sheaves obtained by different choices of subdivisions $\widetilde{\Delta}$ are canonically isomorphic. This somewhat surprising fact can be understood as follows. Let $\mathcal{F}=\phi_{*} \mathcal{A}_{\widetilde{\Delta}}$. If $\sigma \in \Delta$ is a 3 -cone, then the multiplication map

$$
V^{*} \otimes \mathcal{F}(\sigma)_{1} \rightarrow \mathcal{F}(\sigma)_{2}
$$

is surjective. This can be checked directly, or else deduced from Theorem 2.6, which implies that ${\overline{\mathcal{F}(\sigma)_{2}}}_{2}=0$. Thus classes in $\mathcal{F}(\sigma)_{2}$ can be specified by choosing a preimage in $V^{*} \otimes \mathcal{F}(\sigma)_{1}$, which is clearly independent of the chosen subdivision.

Thus $\mathcal{L}(\Delta)_{2}$ is identified with degree two conewise polynomial functions on the simplicial fan $\widetilde{\Delta}_{\leq 3}$. As we noted earlier, sections of $\mathcal{A}$ on a simplicial fan gives the face ring of the corresponding abstract simplicial complex. The decomposition of $\mathcal{L}(\Delta)_{1}$ by the monomial generators is just (10); the corresponding decomposition of $\mathcal{L}(\Delta)_{2}$ by monomials gives an identification

$$
\mathcal{L}(\Delta)_{2}=\bigoplus_{\rho_{1} \triangleleft \rho_{2}}\left\langle\rho_{1}\right\rangle \otimes\left\langle\rho_{2}\right\rangle
$$

where the relation $\rho_{1} \triangleleft \rho_{2}$ for $\rho_{1}, \rho_{2} \in \Delta_{1}$ means that either $\rho_{1}=\rho_{2}$ or else $\rho_{1}$ and $\rho_{2}$ generate a two-dimensional cone of $\widetilde{\Delta}$ and $\rho_{1}$ precedes $\rho_{2}$ in some fixed total order on $\Delta_{1}$.

The quotient space $\overline{\mathcal{L}(\Delta)_{2}}$ is the cokernel of the multiplication map $\mu_{2}: V^{*} \otimes$ $\mathcal{L}(\Delta)_{1} \rightarrow \mathcal{L}(\Delta)_{2}$. In terms of (10) and (12), this map is

$$
\bigoplus_{\rho \in \Delta_{1}} V^{*} \otimes\langle\rho\rangle \rightarrow \bigoplus_{\rho_{1} \triangleleft \rho_{2}}\left\langle\rho_{1}\right\rangle \otimes\left\langle\rho_{2}\right\rangle,
$$

where $V^{*} \otimes\langle\rho\rangle$ maps to all terms on the right of the form $\langle\rho\rangle \otimes\left\langle\rho^{\prime}\right\rangle$ or $\left\langle\rho^{\prime}\right\rangle \otimes\langle\rho\rangle$ via the natural map $V^{*} \rightarrow\left\langle\rho^{\prime}\right\rangle$.

Unlike the discussion of $\mu_{1}$ in the previous section, $\mu_{2}$ is never injective, because $\mathcal{L}(\Delta)$ has a generator in degree zero. Writing down the "obvious" elements in 
the kernel of $\mu_{2}$ gives rise to a two-step chain complex

$$
\bigwedge^{2} V^{*} \stackrel{\phi}{\longrightarrow} \bigoplus_{\rho \in \Delta_{1}} V^{*} \otimes\langle\rho\rangle \stackrel{\mu_{2}}{\longrightarrow} \bigoplus_{\rho_{1} \triangleleft \rho_{2}}\left\langle\rho_{1}\right\rangle \otimes\left\langle\rho_{2}\right\rangle,
$$

where

$$
\phi(\alpha \wedge \beta)=\alpha \otimes \mu_{1}(\beta)-\beta \otimes \mu_{1}(\alpha)
$$

for any $\alpha, \beta \in V^{*}$. In other words, $\mu_{2} \circ \phi=0$.

Now suppose that $\Delta$ is either the central fan $\Delta_{P}$ or the cone $[c P]$ for some polytope $P$. over a polytope of dimension $d-1$. Then the complex $(13)$ is left exact; i.e., it has cohomology only at the rightmost place. The injectivity of $\phi$ follows from the fact that $\Delta$ contains a full-dimensional cone, since then $\phi$ factors through the natural map $\bigwedge^{2} V^{*} \rightarrow V^{*} \otimes V^{*}$. Exactness in the middle follows from the fact that $\mathcal{L}(\Delta)$ is a free $A$-module in positive degrees with a single generator in degree zero. Note that this depends on the identifications (10) and (12), which in turn required the use of Theorem 2.6.

2.9. Connection with infinitesimal rigidity. Conversely, knowing the exactness of (13) when $\Delta=[\sigma]$ for $\sigma$ a cone of dimension $d>3$ implies, without appealing to Theorem 2.6, that the restriction $\mathcal{L}(\sigma)_{2} \rightarrow \mathcal{L}(\partial \sigma)_{2}$ is an isomorphism, and thus by induction that $\mathcal{L}(\sigma)_{2} \rightarrow \mathcal{L}\left(\Delta_{\leq 3}\right)_{2}$ is an isomorphism, which in turn implies the formula (12). As we will see, this exactness can be deduced from results of Whiteley about infinitesimal rigidity of frameworks. So the theory up to degree two can be verified independently, without using Karu's theorem.

To see the rigidity interpretation of (13), we replace it by a smaller but equivalent subcomplex. Both the source and target of $\mu_{2}$ surject onto $\mathcal{L}\left(\Delta_{\leq 1}\right)_{2}=$ $\bigoplus_{\rho \in \Delta_{1}}\langle\rho\rangle \otimes\langle\rho\rangle$ via maps commuting with $\mu_{2}$. Taking kernels gives a smaller complex with the same cohomology:

$$
0 \rightarrow \bigwedge^{2} V^{*} \rightarrow \bigoplus_{\rho \in \Delta_{1}} \rho^{\perp} \otimes\langle\rho\rangle \stackrel{\tilde{\mu}_{2}}{\longrightarrow} \bigoplus_{\substack{\rho_{1} \triangleleft \rho_{2} \\ \rho_{1} \neq \rho_{2}}}\left\langle\rho_{1}\right\rangle \otimes\left\langle\rho_{2}\right\rangle,
$$

where $\rho^{\perp} \subset V^{*}$ is the annihilator of $\operatorname{span}(\rho)$.

Now suppose that $\Delta=[c P]$ is the cone over a $d$-polytope $P$ in a vector space $W$, so that $\Delta$ is a fan in $V=W \oplus \mathbb{R}$. The dimensions of the terms of (14) of $P$ are $\left(\begin{array}{c}d+1 \\ 2\end{array}\right), d f_{0}$, and $f_{1}+\left(f_{02}-3 f_{2}\right)$, respectively. Thus the formula (6) for $g_{2}(P)$ computes the Euler characteristic of this complex. Since the complex has cohomology only at the rightmost term, we see that $g_{2}(P)$ is the dimension of $\operatorname{coker}\left(\tilde{\mu}_{2}\right) \cong \operatorname{coker}\left(\mu_{2}\right)={\overline{\mathcal{L}}(\Delta)_{2}}_{2}$, as asserted by Theorem 2.4.

The fan $\Delta_{\leq 2}$ is the cone over a framework, or geometric graph, whose vertices are the vertices of $P$ and whose edges are the edges of $P$ together with enough extra edges to triangulate each 2-face. A 1-cone $\rho \in \Delta_{1}$ will be the cone over a 
vector $(v, 1)$, where $v \in V$ is a vertex of $P$. This gives an identification $\langle\rho\rangle \cong \mathbb{R}$ via $\phi \mapsto \phi(v, 1)$. By restricting covectors we get an identification of $\rho^{\perp} \subset(W \oplus \mathbb{R})^{*}$ with $W^{*}$, and choosing an inner product on $W$, we get an identification $\rho^{\perp} \cong W$.

With these identifications the map $\tilde{\mu}_{2}$ on the right of (14) becomes the infinitesimal rigidity matrix of the framework, which is a linear map

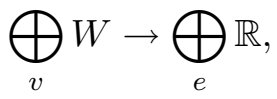

where the sums are over all vertices $v$ and edges $e$ of the framework. It sends a tuple $\left(w_{v}\right)$ to $\left(w_{v}-w_{v^{\prime}}\right) \cdot\left(v-v^{\prime}\right)$ on an edge $e$ with endpoints $v, v^{\prime}$. Elements of $\bigoplus_{v} W$ are assignments of vectors to each vertex of $P$ which should be thought of as infinitesimal motions of these points. Being in the kernel of $\mu_{2}$ means that the lengths of the edges of $P$ do not change to first order under this motion, with respect to the chosen inner product.

The image of $\bigwedge^{2} V^{*}$ in $\bigoplus_{v} W$ gives assignments of vectors which come from global affine motions of $W$. Thus the exactness of (13) translates to the statement that the framework is infinitesimally rigid - all motions of the vertices preserving the lengths of the edges to first order come from global affine motions. When $\operatorname{dim} P=3$, this was proved by Aleksandrov, generalizing theorems of Cauchy and Dehn for the case when $P$ is simplicial (see also [FP, P]). In higher dimensions, Whiteley $[\mathrm{W}]$ showed how to deduce rigidity inductively from the three-dimensional case.

The cohomology of the dual complex to (14) is known as the space of stresses of the framework; these can be viewed as assignments of expanding or contracting forces along the edges in such a way that the total forces at each vertex cancel. Before the advent of combinatorial intersection cohomology, Kalai [Kal1, Kal3] pointed out that this gives a vector space of dimension $g_{2}(P)$, thus proving $g_{2}(P) \geq 0$. He also used the interpretation of $g_{2}$ as a space of stresses to prove the degree two piece of his monotonicity conjecture: $g_{2}(P) \geq$ $g_{2}(F)+g_{1}(F) g_{1}(P / F)+g_{2}(P / F)$.

Several authors [L, L2, TWW1, TWW2] have considered a theory of "higher rigidity" for simplicial complexes which plays the same role for higher $g_{k}$ 's that infinitesimal rigidity plays for $g_{2}$. These theories are closely related to McMullen's polytope algebra.

This connection between $g_{2}$ and rigidity explains why Theorems 2.5 and 2.6 depend on the inductive nature of fans (they are made up of cones over convex polytopes, which can be described by polytopal fans in one dimension less, and so on). Connelly [C1, C2] has constructed non-convex simplicial triangulated spheres in $\mathbb{R}^{3}$ which are not rigid. The cone over such a sphere will be a 3dimensional fan in $\mathbb{R}^{4}$; if this is considered to be the boundary of a non-convex "cone", then the failure of exactness of the rigidity complex means that Theorem 
2.4 does not hold. The degree vanishing and rigidity (Theorems 2.6, 2.7) also fail.

It would be interesting to find a relation between combinatorial IH and Sabitov's result $[\mathrm{S}]$ that the volume of a flexible triangulated 3-sphere remains constant as it flexes.

\section{New inequalities}

As with any sheaf theory, combinatorial IH sheaves give rise to a wide variety of homomorphisms, complexes and exact sequences. We present two applications which produce new inequalities among the $h$ - and $g$-numbers. The first answers a question of Stanley from [St2] and the second generalizes Kalai's monotonicity (Theorem 1.2) specialized at $t=1$.

3.1. Decompositions of $h(P, t)$ from a shelling. Let $P$ be a $d$-polytope. The sum (4) which gives the $g$-polynomial of $P$ runs over all faces in the boundary complex $\partial P$, which is a polyhedral subdivision of a $(d-1)$-sphere. In this section, we consider replacing (4) by partial sums over subsets of $\partial P$. More specifically, let $I$ be a subcomplex of $\partial P$, and let $J$ be a subcomplex of $I$. Then we define

$$
h(I, J, t)=\sum_{F \in I \backslash J} g(F, t)(t-1)^{d-1-\operatorname{dim} F} .
$$

This definition is considered in [St2] under the more general hypothesis that $I$ is a locally Eulerian poset and $J$ is an order ideal in $I$.

If we have a filtration $\emptyset=I_{0} \subset I_{1} \subset \cdots \subset I_{r}=\partial P$ by subcomplexes, we therefore get a decomposition

$$
h(P, t)=\sum_{j=1}^{r} h\left(I_{j}, I_{j-1}, t\right) .
$$

If the polynomials $h\left(I_{j}, I_{j-1}\right)$ have nonnegative coefficients, this will result in inequalities for the $h$-numbers of $P$.

The following result answers a question of Stanley [St2, Section 6]. Suppose that we are given a shelling of $\partial P$; i.e. an ordering $F_{1}, \ldots, F_{r}$ of the facets of $P$ such that for every $k=1, \ldots, r-1$ the union $I_{k}=F_{1} \cup \cdots \cup F_{k}$ is topologically a $(d-1)$-dimensional disk. Boundary complexes of polytopes can always be shelled, for instance by the line shelling construction of Bruggesser and Mani [BruM].

Theorem 3.1. For $1 \leq j \leq r$, the coefficients of $h\left(I_{j}, I_{j-1}, t\right)$ are nonnegative.

Note that this polynomial is "locally defined", in the sense that it depends only on $F_{j}$ and its intersection with $\bigcup_{i<j} F_{i}$. Thus Theorem 3.1 implies that the $h$-numbers are nonnegative for shellable polyhedral complexes. 
It is elementary to see that the sum of the coefficients of $h\left(I_{j}, I_{j-1}, t\right)$ is nonnegative, since setting $t=1$ in $(16)$ gives $h\left(I_{j}, I_{j-1}, 1\right)=g\left(F_{j}, 1\right)$. One case where it is easy to see that the individual coefficients are nonnegative is when $F_{j}$ is a simplex, which gives $h\left(I_{j}, I_{j-1}, t\right)=t^{k}$, where $k$ is the number of facets of $F_{j}$ not in $\bigcup_{i<j} F_{i}$. Another is when $j=1$ and $j=r$, which gives

$$
\begin{aligned}
h\left(I_{1}, \emptyset, t\right) & =t^{d} g\left(F_{1}, t^{-1}\right) \\
h\left(I_{r}, I_{r-1}, t\right) & =g\left(F_{r}, t\right) .
\end{aligned}
$$

The nonnegativity for other $j$ is new, although Bayer [Bay2] showed nonnegativity for certain shellings of a class of nonsimplicial polytopes generalizing cyclic polytopes.

Example. Let $P$ be a prism over a 2 -simplex, and take any shelling $F_{1}, \ldots, F_{5}$ for which $F_{1}$ and $F_{4}$ are the simplicial facets. Then

$$
\begin{aligned}
h\left(I_{1}, \emptyset, t\right) & =t^{3} \\
h\left(I_{2}, I_{1}, t\right) & =2 t^{2} \\
h\left(I_{3}, I_{2}, t\right) & =t+t^{2} \\
h\left(I_{4}, I_{3}, t\right) & =t \\
h\left(I_{5}, I_{4}, t\right) & =1+t
\end{aligned}
$$

and $h(P, t)=t^{3}+2 t^{2}+\left(t+t^{2}\right)+t+(1+t)=1+3 t+3 t^{2}+t^{3}$.

The proof of Theorem 3.1 relies on the following beautiful result of Barthel, Brasselet, Fieseler, and Kaup, which characterizes when $\mathcal{L}(\Delta)$ is a free $A$-module. Let $\Delta$ be a purely $d$-dimensional fan in a $d$-dimensional vector space, and let $\partial \Delta$ denote the subfan generated by the $(d-1)$-dimensional cones which are contained in exactly one $d$-dimensional cone of $\Delta$.

Theorem 3.2 ([BBFK2]). $\mathcal{L}(\Delta)$ is a free A-module if and only if $\partial \Delta$ is an $\mathbb{R}$-homology manifold. If this holds, then

$$
\operatorname{Hilb}(\overline{\mathcal{L}(\Delta)}, t)=\sum_{\sigma \in \Delta \backslash \partial \Delta} g(\sigma, t)(t-1)^{d-\operatorname{dim} \sigma},
$$

where we put $g(\sigma, t)=g(F, t)$ when $\sigma=c F$, the cone over a polytope $F$.

Following [BBFK2], we call fans satisfying these equivalent conditions quasiconvex. Examples of quasi-convex fans include complete fans (where $\partial \Delta$ is empty) and full-dimensional cones (where $\partial \Delta$ is homeomorphic to $\mathbb{R}^{d-1}$ ).

Proof of Theorem 3.1. Let $\Delta=\Delta_{P}$ be the central fan of the $d$-polytope $P$, and let $\sigma_{1}, \ldots, \sigma_{r}$ be the ordering on the top-dimensional cones of $\Delta$ given by taking cones over the $F_{i}$. 
Let $\Delta^{j}=\left[\sigma_{j}\right] \cup \cdots \cup\left[\sigma_{r}\right]$ be the fan generated by $\sigma_{j}, \ldots, \sigma_{r}$. Then $\partial \Delta^{j}$ is the cone over $\partial\left(F_{j} \cup \cdots \cup F_{s}\right)=\partial\left(I_{j-1}\right)$, which is a $(d-1)$-sphere since the ordering of the $F_{i}$ is a shelling. Thus $\Delta^{j}$ is quasi-convex. By Theorem 3.2, we have

$$
\operatorname{Hilb}\left(\overline{\mathcal{L}\left(\Delta^{j}\right)}, t\right)=\sum_{F \notin I_{j-1}} g(F, t)(t-1)^{d-1-\operatorname{dim} F} .
$$

Now for $1 \leq j \leq r$ consider the restriction $\mathcal{L}\left(\Delta^{j}\right) \rightarrow \mathcal{L}\left(\Delta^{j+1}\right)$, where we set $\Delta^{r+1}=\emptyset$. It is a surjective map of free $A$-modules, which implies that its kernel $K$ is also free. It follows that

$$
\begin{aligned}
\operatorname{Hilb}(\bar{K}, t) & =\operatorname{Hilb}\left(\overline{\mathcal{L}\left(\Delta^{j}\right)}, t\right)-\operatorname{Hilb}\left(\overline{\mathcal{L}\left(\Delta^{j+1}\right)}, t\right) \\
& =\sum_{F \in I_{j} \backslash I_{j-1}} g(F, t)(t-1)^{d-1-\operatorname{dim} F} \\
& =h\left(I_{j}, I_{j-1}, t\right) .
\end{aligned}
$$

Thus the coefficients of $h\left(I_{j}, I_{j-1}, t\right)$ are nonnegative.

Remark. This result can also be deduced by applying Proposition 6.7 of [BBFK2] to the pair of fans $\left(\left[\sigma_{i}\right],\left[\sigma_{i}\right] \cap \Delta_{i-1}\right)$.

When $\Delta=\Delta_{P}$ for a rational polytope $P$ in $V$, and the shelling of $P$ is a line shelling, the local $h$-polynomials can be understood in terms of a topological construction. The line shelling depends on the choice of a vector $v \in V$; if this vector is in the lattice, it determines a homomorphism from $\mathbb{C}^{*} \rightarrow T$ and thus an action of $\mathbb{C}^{*}$ on the toric variety $X_{\Delta}$. The condition that this direction determines a shelling ensures that the action has isolated fixed points.

The resulting flow on $X_{\Delta}$ induces a partial order on the set $X_{\Delta}^{T}$ of fixed points. The fixed points are in bijection with the facets of $P$, and the partial order is compatible with the total order from the shelling. The decomposition (17) then comes from a result of Kirwan [Kir] which gives a decomposition of the equivariant intersection cohomology of a $T$-variety $X$ into a sum of terms coming from each component of the locus of points fixed by a one-dimensional subtorus of $T$.

3.2. A generalization of Kalai's monotonicity at $t=1$. Our next application gives a lower bound for $g(P, 1)=\sum g_{i}(P)$ which generalizes Theorem 1.2 specialized to $t=1$. For the following discussion it will be convenient to work entirely with cones instead of polytopes; if $\sigma$ is the cone $c P$ over a compact polytope $P$, we put $g(\sigma, t)=g(P, t)$.

Fix a full-dimensional cone $\sigma$ in a vector space $V$, and let $\Delta=[\sigma]$. Fix a vector $v \in V$, and let $\Delta_{0}$ denote the set of faces $\tau \in \Delta$ for which $v \in \operatorname{span}(\tau)$. 
Theorem 3.3. Let Min $\Delta_{0}$ be the set of minimal faces in $\Delta_{0}$. Then

$$
g(\sigma, 1) \geq \sum_{\tau \in \operatorname{Min} \Delta_{0}} g(\tau, 1) g(\sigma / \tau, 1) .
$$

Unlike the other results described so far, this is not a purely combinatorial statement about face posets of cones and polytopes, since in general the possible sets Min $\Delta_{0}$ will depend on the angles of the faces with respect to $v$, and not just their inclusion relations. There is one case where $\Delta_{0}$ can be determined combinatorially, however: when $v$ lies in the relative interior of a face $\tau$ of $\sigma$, then $\Delta_{0}=\operatorname{st}(\tau)$. Therefore Min $\Delta_{0}=\{\tau\}$, so Theorem 3.3 becomes

$$
g(\sigma, 1) \geq g(\tau, 1) g(\sigma / \tau, 1) .
$$

If $\sigma=c P$ and $\tau=c F$ for a polytope $P$ and a face $F$ of $P$, then $\sigma / \tau=c(P / F)$, and we recover Theorem 1.2 at $t=1$.

Example. Let $\sigma$ be the cone over a square, and choose $v$ so that $\Delta_{0}$ contains two opposite 2-faces $\tau_{1}, \tau_{2}$ in addition to $\sigma$ itself. Then $\operatorname{Min} \Delta_{0}=\left\{\tau_{1}, \tau_{2}\right\}$, and Theorem 3.3 says that

$$
g(\sigma, 1) \geq \sum_{i=1,2} g\left(\tau_{i}, 1\right) g\left(\sigma / \tau_{i}, 1\right)=1 \cdot 1+1 \cdot 1=2 .
$$

In this case equality holds, since $g(\sigma, t)=1+t$. Note that this example shows that evaluating at $t=1$ is necessary.

To prove Theorem 3.3, we restrict the combinatorial IH sheaf $\mathcal{L}_{\Delta}$ from $\Delta$ to $\Delta_{0}$ in a way which uses the direction of the vector $v$. It is a combinatorial counterpart of the "hyperbolic localization" of [B]. In order to describe it, decompose the fan $\Delta=[\sigma]$ according to its intersection with lines parallel to $v$.

Definition 3.4. Call a face $\tau \in \Delta$ a back face if for every point $x \in \tau^{\circ}$, the set

$$
\{t \in \mathbb{R} \mid x+t v \in \sigma\}
$$

contains some interval $\left[0, \epsilon_{x}\right), \epsilon_{x}>0$. Let $\Delta_{\leq 0}$ be the set of all back faces. Using the same definitions but with $v$ replaced by $-v$, define a subset $\Delta_{\geq 0} \subset \Delta$ of front faces.

It is easy to see that $\Delta_{0}=\Delta_{\leq 0} \cap \Delta_{\geq 0}$. We also put $\Delta_{+}=\Delta_{\geq 0} \backslash \Delta_{0}$, and $\Delta_{-}=\Delta_{\leq 0} \backslash \Delta_{0}$.

If $\tau \in \Delta_{\leq 0}$ is a back face, then there is a unique face $\rho \prec \sigma$ so that $x+t v \in \rho^{\circ}$ for any $x \in \tau^{\circ}$ and any $t \in\left(0, \epsilon_{x}\right)$; we denote this face by $\tau_{+v}$. Note that if $\tau \in \Delta_{0}$, then $\tau_{+v}=\tau$.

Proposition 3.5. These sets satisfy the following:

(1) $\Delta_{\geq 0}, \Delta_{\leq 0}$, and $\Delta_{0}$ are closed in the fan topology on $\Delta$. 
(2) $\tau \mapsto \tau_{+v}$ is a surjective function $\Delta_{\leq 0} \rightarrow \Delta_{0} ; \tau_{+v}$ is the unique smallest face in $\Delta_{0}$ with $\tau \prec \tau_{+v}$.

(3) $\left[\Delta_{+}\right]=\Delta \backslash \Delta_{\leq 0}$

(4) The projection $p: V \rightarrow V / \mathbb{R} v$ gives a conewise linear isomorphism between $\left[\Delta_{+}\right]$and a fan $p_{*}\left[\Delta_{+}\right]$with support $p(|\Delta|)=p(\sigma)$.

The last two statements can be understood as follows: the subfan $\left[\Delta_{+}\right]$consists of all faces of $\sigma$ illuminated by light shining from infinity in parallel beams with direction $-v$.

Given an arbitrary subset $\Sigma \subset \Delta$, define $\Sigma_{\geq 0}=\Delta_{\geq 0} \cap \Sigma$ and $\Sigma_{+}=\Delta_{+} \cap \Sigma$.

Definition 3.6. Given a $\mathcal{A}_{\Delta}$-sheaf $\mathcal{F}$, its $v$-localization is the sheaf $\mathcal{F}^{v}$ defined by

$$
\mathcal{F}^{v}(\Sigma)=\mathcal{F}\left(\left[\Sigma_{\geq 0}\right],\left[\Sigma_{+}\right]\right)
$$

for any subfan $\Sigma$ of $\Delta$.

The support of $\mathcal{F}^{v}$ is contained in $\Delta_{0}$, since if $\Sigma \cap \Delta_{0}=\emptyset$, then $\Sigma_{\geq 0}=\Sigma_{+}$, and so $\mathcal{F}^{v}(\Sigma)=0$.

Theorem 3.7. If $\mathcal{F}$ is a pure $\mathcal{A}_{\Delta}$-sheaf, then $\mathcal{F}^{v}$ is also pure.

Proposition 3.8. For every $\tau \in \Delta_{0}$, we have $\operatorname{dim}_{\mathbb{R}} \overline{\mathcal{F}^{v}(\tau)}=\operatorname{dim}_{\mathbb{R}} \overline{\mathcal{F}(\tau)}$.

Note that the Hilbert polynomials of these modules are not in general equal; it is essential here to ignore the grading and evaluate at $t=1$.

We now deduce Theorem 3.3 from these results. Let $\mathcal{L}={ }_{o} \mathcal{L}$. By Theorem 3.7 $\mathcal{L}^{v}$ is a direct sum of shifted copies of sheaves ${ }_{\tau} \mathcal{L}, \tau \in \Delta$. Since the support of $\mathcal{L}^{v}$ lies in $\Delta_{0}$, we have

$$
\mathcal{L}^{v} \cong \bigoplus_{\tau \in \Delta_{0}}{ }_{\tau} \mathcal{L} \otimes N_{\tau}
$$

where the $N_{\tau}$ are graded $\mathbb{R}$-vector spaces.

Proposition 3.8 implies that $\operatorname{dim}_{\mathbb{R}} N_{\tau}=\operatorname{dim}_{\mathbb{R}} \overline{\mathcal{L}(\tau)}=g(\tau, 1)$ if $\tau \in \operatorname{Min} \Delta_{0}$. By taking just those terms in (19) with $\tau \in \operatorname{Min} \Delta_{0}$, we get

$$
g(\sigma, 1)=\operatorname{dim}_{\mathbb{R}} \mathcal{L}^{v}(\tau) \geq \sum_{\tau \in \operatorname{Min} \Delta_{0}} \operatorname{dim}_{\mathbb{R}}(\overline{(\tau \mathcal{L}(\sigma)}) \operatorname{dim}_{\mathbb{R}}\left(N_{\tau}\right)=\sum_{\tau \in \operatorname{Min} \Delta_{0}} g(\tau, 1) g(\sigma / \tau, 1),
$$

which is Theorem 3.3.

Note that when $v$ lies in the relative interior of a cone $\tau \prec \sigma$, we have $\Delta_{\geq 0}=\operatorname{st}(\tau), \Delta_{+}=\emptyset$, and so $\mathcal{L}^{v}=\left.\mathcal{L}\right|_{\operatorname{st}(\tau)}$. The same argument then works without appealing to Proposition 3.8, and in fact gives a graded statement without evaluating at $t=1$. There is only one term in (18), since Min $\Delta_{0}=\{\tau\}$. This is precisely the argument in [BBFK2, BrL1] used to prove Theorem 1.2. 
3.3. Proofs. We give the proofs of Theorem 3.7 and Proposition 3.8.

First we show that if $\mathcal{F}$ is a flabby sheaf, then $\mathcal{F}^{v}$ is again a flabby sheaf. We use the following characterization of flabby sheaves. Note that flabbiness of an $\mathcal{A}_{\Delta}$-module only depends on its structure as a sheaf of graded vector spaces, and not the module structure. For a cone $\tau \in \Delta$, let $\underline{\mathbb{R}}_{\mathrm{st}(\tau)}$ be the constant sheaf on $\operatorname{st}(\tau)$ with stalk $\mathbb{R}$, extended by zero to all of $\Delta$. Then $\underline{\mathbb{R}}_{\mathrm{st}(\tau)}(\Sigma)=\mathbb{R}$ if $\tau \in \Sigma$ and is 0 if $\tau \notin \Sigma$, and the restriction maps are the identity whenever possible. The following lemma is essentially Lemma 3.6 from [BBFK2].

Lemma 3.9. A sheaf of graded vector spaces on $\Delta$ is flabby if and only if it is a direct sum of sheaves $\underline{\mathbb{R}}_{\mathrm{st}(\tau)}$, $\tau \in \Delta$, with shifts.

Using this, the following lemma shows that if $\mathcal{F}$ is flabby then $\mathcal{F}^{v}$ is flabby.

Lemma 3.10. The v-localization of $\mathcal{F}=\underline{\mathbb{R}}_{\mathrm{st}(\tau)}$ is given by

$$
\mathcal{F}^{v} \cong \begin{cases}\mathbb{R}_{\mathrm{st}(\tau+v)}, \tau \in \Delta_{\leq 0} \\ 0 & \text { otherwise. }\end{cases}
$$

Proof. If $\Sigma$ is a subfan of $\Delta$, then

$$
\mathcal{F}^{v}(\Sigma)=\left\{\begin{array}{l}
\mathbb{R}, \tau \in\left[\Sigma_{\geq 0}\right] \backslash\left[\Sigma_{+}\right] \\
0 \text { otherwise }
\end{array}\right.
$$

Suppose first that $\tau \in\left[\Sigma_{\geq 0}\right] \backslash\left[\Sigma_{+}\right]$. Then there exists $\rho \in \Delta_{\geq 0} \backslash \Delta_{+}=\Delta_{0}$ with $\tau \prec \rho$. If $\tau$ were in $\left[\Delta_{+}\right]$then there would be a cone $\nu \in \Delta_{+}$with $\tau \prec \nu \prec \rho$. This is because the projection $p(\rho)$ is a union of cones containing $\tau$ in the projected fan $p_{*}\left[\Delta_{+}\right]$, so one can take $\nu$ to be a cone which projects to a maximal-dimensional cone contained in $p(\rho)$ and containing $p(\tau)$. But this means that $\nu \in[\rho] \subset \Sigma$, so $\tau \in\left[\Sigma_{+}\right]$, contrary to assumption. So $\tau \in \Delta_{0} \backslash\left[\Delta_{+}\right]=\Delta_{\leq 0}$. Then part (2) of Proposition 3.5 implies that $\tau_{+v} \prec \rho$, so $\Sigma \cap \operatorname{st}\left(\tau_{+v}\right) \neq \emptyset$.

Conversely, if $\tau \in \Delta_{\leq 0}$ and $\Sigma \cap \operatorname{st}\left(\tau_{+v}\right) \neq \emptyset$, then in particular $\tau_{+v} \in \Sigma \cap \Delta_{0}=$ $\Sigma_{0}$, so $\tau \in\left[\Sigma_{\geq 0}\right] \backslash\left[\Sigma_{+}\right]$.

Next we show that $\mathcal{F}^{v}$ is locally free if $\mathcal{F}$ is pure. Take a cone $\tau \in \Delta_{0}$. Then $[\tau]_{\geq 0}=[\tau] \cap \Delta_{\geq 0}$ is the set of front faces of $\tau$, considered as a cone in $\operatorname{span}(\tau)$, with respect to the vector $v$, and similarly for the back faces. So to show that $\mathcal{F}^{v}$ is locally free, it is enough to show that the stalk at $\sigma$

$$
\mathcal{F}^{v}([\sigma])=\mathcal{F}\left(\left[\Delta_{\geq 0}\right],\left[\Delta_{+}\right]\right)
$$

is a free $A$-module. This will follow from the following result and Proposition $3.5(4)$.

Theorem 3.11. Let $\sigma$ be a full-dimensional cone in $V$, and let $\mathcal{F}$ be a pure $\mathcal{A}_{[\sigma]}$-module. Suppose that $\Sigma$ is a purely $(d-1)$-dimensional subfan of $\partial \sigma$ which 
is conewise linearly isomorphic to a quasi-convex fan via the projection $p: V \rightarrow$ $V / \mathbb{R} v$ for some $v \in V$. Then

$\mathcal{F}([\sigma], \Sigma)$ is a free $A$-module, of the same total rank as $\mathcal{F}(\sigma)$.

Proof. An $A$-module $M$ is free if and only if $\operatorname{Tor}_{i}^{A}(M, \mathbb{R})=0$ for $i>0$ (see $[\mathrm{BBFK} 2, \S 0 . \mathrm{B}])$. Using the long exact Tor sequence of

$$
0 \rightarrow \mathcal{F}([\sigma], \Sigma) \rightarrow \mathcal{F}([\sigma]) \rightarrow \mathcal{F}(\Sigma) \rightarrow 0
$$

and the freeness of $\mathcal{F}([\sigma])$, we are reduced to showing that $\operatorname{Tor}_{i}^{A}(\mathcal{F}(\Sigma), \mathbb{R})=0$ for $i>1$ and that $\operatorname{dim} \operatorname{Tor}_{0}^{A}(\mathcal{F}(\Sigma))=\operatorname{dim} \operatorname{Tor}_{1}^{A}(\mathcal{F}(\Sigma))$.

To see this, let $\hat{\Sigma}$ denote the fan in $\hat{V}=V / \mathbb{R} v$ obtained by projecting $\Sigma$ by $p$. Also let $\hat{A}$ denote the ring of polynomial functions on $V / \mathbb{R} v$; the pullback $p^{*}: \hat{A} \rightarrow A$ makes it into a subring of $A$.

Since $p$ is a conewise linear isomorphism, pushing forward the restriction of $\mathcal{F}$ to $\Sigma$ gives a pure sheaf on $\hat{\Sigma}$ whose global sections are just $\mathcal{F}(\Sigma)$, with the $\hat{A}$-module structure induced by $p^{*}$. This means that $\mathcal{F}(\Sigma)$ is a free $\hat{A}$-module, by Theorem 3.2.

Let $M=\mathcal{F}(\Sigma)$. Then the $A$-module structure is determined by the $\hat{A}$-module structure together with the homomorphism $M \rightarrow M$ of multiplication by $y$, where $y$ is any element in $A_{1}$ not in $\hat{A}_{1}$.

There is a short exact sequence

$$
0 \rightarrow A \otimes_{\hat{A}} M \rightarrow A \otimes_{\hat{A}} M \rightarrow M \rightarrow 0
$$

of $A$-modules, where the first map is $a \otimes m \mapsto a \otimes y m-y a \otimes m$ and the second map is $a \otimes m \mapsto a m$. The first two terms of this sequence give a free resolution of $M$ as an $A$-module, and tensoring with $\mathbb{R}$ gives a finite-dimensional two-step complex $M \otimes_{\hat{A}} \mathbb{R} \rightarrow M \otimes_{\hat{A}} \mathbb{R}$ whose homology in degree $i$ is $\operatorname{Tor}_{i}^{A}(M, \mathbb{R})$. The theorem follows.

\section{Polytope duality and Stanley's convolution identity}

As a final application, we explain how an identity of Stanley relating the $g$ numbers of a polytope $P$ and its polar $P^{*}$ can be understood in the combinatorial $\mathrm{IH}$ language. We also discuss how this is related to the toric Koszul duality constructed in $[\mathrm{BL}]$.

For a polytope $P$ which spans the linear space $V$, the polar polytope is

$$
P^{*}=\left\{w \in V^{*} \mid\langle v, w\rangle \geq-1 \text { for all } v \in P\right\},
$$


where $P$ is translated so that 0 lies in its interior; it is well-defined up to projective equivalence. There is an order-reversing inclusion between the face lattices of $P$ and $P^{*}$.

In [St3], Stanley showed that for any polytope $P \neq \emptyset$ we have

$$
\sum_{\emptyset \leq F \leq P}(-1)^{\operatorname{dim} F} g\left(F^{*}, t\right) g(P / F, t)=0 .
$$

In particular, if $\operatorname{dim} P=2 k$ is even, then the degree $k$ piece of this identity gives $g_{k}(P)=g_{k}\left(P^{*}\right)$. In fact $(20)$ holds for general Eulerian posets, but our interpretation will only be valid for polytopes.

We will show that Stanley's formula can be "lifted" to a statement in linear algebra, by exhibiting a long exact sequence of graded vector spaces whose graded Euler characteristic is the alternating sum in (20). It is obtained by taking stalk cohomology of a certain complex of $\mathcal{A}_{\Delta}$-modules. A more detailed discussion of the homological algebra of $\mathcal{A}_{\Delta}$-modules appears in [BL, Section 6], but for our purposes it is enough to remark that a sequence $0 \rightarrow \mathcal{E} \rightarrow \mathcal{F} \rightarrow \mathcal{G} \rightarrow 0$ of $\mathcal{A}_{\Delta^{-}}$ modules is short exact if and only if the induced sequence on stalks $0 \rightarrow \mathcal{E}(\sigma) \rightarrow$ $\mathcal{F}(\sigma) \rightarrow \mathcal{G}(\sigma) \rightarrow 0$ is a short exact sequence of $A_{\sigma}$-modules for every $\sigma \in \Delta$.

The complex we want is described by the following theorem. Fix a fan $\Delta$ and let $r=\operatorname{dim} \Delta$ be the dimension of its largest cone. Let $\mathcal{M}$ be the $\mathcal{A}_{\Delta}$-module which is the extension by zero of the rank one constant sheaf on $\{o\}$; in other words, we have $\mathcal{M}(o)=\mathbb{R}$ and $\mathcal{M}(\tau)=0$ for $\tau \neq o$.

Theorem 4.1. There exists a resolution

$$
0 \rightarrow \mathcal{M} \rightarrow \mathcal{F}^{0} \rightarrow \mathcal{F}^{1} \rightarrow \cdots \rightarrow \mathcal{F}^{r} \rightarrow 0
$$

of $\mathcal{M}$ by pure sheaves so that $\mathcal{F}^{0}={ }_{0} \mathcal{L}_{\Delta}$ and for $i>0 \mathcal{F}^{i}$ is a direct sum of sheaves of the form ${ }_{\tau} \mathcal{L}_{\Delta}\left[-\frac{\operatorname{dim} \tau-i}{2}\right]$, with $\operatorname{dim} \tau-i \in 2 \mathbb{Z}_{\geq 0}$; it is unique up to a unique automorphism, after fixing a basis of $\mathcal{M}(0)=\mathbb{R}$.

We postpone the proof until the end of this section.

We are interested in the multiplicity $m_{k}(\tau)$ of the $\operatorname{sheaf}_{\tau} \mathcal{L}_{\Delta}[-k]$ in $\mathcal{F}^{\operatorname{dim} \tau-2 k}$. Since the complex provided by Theorem 4.1 clearly behaves well under restriction of fans, this multiplicity depends only on $\tau$ and not on the ambient fan $\Delta$. It can be computed recursively as follows. If $\tau \neq o$, the stalk complex $\mathcal{F} \bullet(\tau)$ is an exact sequence of free $A_{\tau}$-modules, so the reduced sequence

$$
0 \rightarrow \overline{\mathcal{F}^{0}(\tau)} \rightarrow \cdots \rightarrow \overline{\mathcal{F}^{r}(\tau)} \rightarrow 0
$$

is exact. Taking the Euler characteristic of the graded piece in degree $k$, we can solve for $m_{k}(\tau)$, given the multiplicities $m_{j}(\rho)$ for all $j \leq k$ and all proper faces $\rho$ of $\tau$. Using (20), we get the following result. 
Proposition 4.2. If $\tau$ is a cone over a polytope $P$, then $m_{k}(\tau)=g_{k}\left(P^{*}\right)$ for all $k \geq 0$.

Thus the graded Euler characteristic of the exact sequence (22) gives Stanley's formula. The dimensions of the individual entries can also be organized into a two-variable polynomial $B(P ; u, v)$ considered in $[\mathrm{BaBo}, \mathrm{BoM}]$ as part of their study of stringy Hodge numbers for hypersurfaces in toric varieties.

As usual, an exact sequence gives rise to inequalities by truncation. Taking the first $s+1$ terms of the degree $k$ piece of (22), we see that

$$
\sum(-1)^{\operatorname{dim} F-s+1} g_{i}\left(F^{*}\right) g_{j}(P / F) \geq 0,
$$

where the sum is over all $i$ and $j$ with $i+j=k$ and all faces $\emptyset \leq F \leq P$ with $\operatorname{dim} F \leq s+2 i-1$. Taking $s=0$ just recovers the inequality $g_{k}(P) \geq 0$, since the only nonzero summand in $(23)$ is $F=\emptyset$. For $k=s=1$, the inequality can be reduced to to $g_{2}+f_{1}-f_{0} \geq(d+1)(d-2) / 2$, where $d=\operatorname{dim} P$. This follows from the nonnegativity of $g_{2}$ and the elementary facts that $f_{0} \geq d+1$ and every vertex is contained in at least $d$ edges. For $d \leq 5$ all the other cases of (23) follow from these two cases. In general we do not know if any of these inequalities are new in dimensions $d \geq 6$.

Since the complex $\mathcal{F}^{\bullet}$ is unique up to a unique isomorphism, we can define a canonical multiplicity space of dimension $m_{k}(\tau)$; let $M_{k}(\tau)$ be the image of the degree $k$ part of the map $\overline{\mathcal{F}^{i}(\tau, \partial \tau)} \rightarrow \overline{\mathcal{F}^{i}(\tau)}$, where $i=\operatorname{dim}(\tau)-2 k$. Then we have a canonical isomorphism

$$
\mathcal{F}^{i} \cong \bigoplus_{\operatorname{dim} \tau=2 k+i} M_{k}(\tau) \otimes_{\mathbb{R} \tau} \mathcal{L}[-k] .
$$

Proposition 4.2 then lifts to the following functorial statement. Recall that the dual cone to a full-dimensional cone $\tau$ in $V$ is

$$
\tau^{\vee}=\left\{y \in V^{*} \mid\langle x, y\rangle \geq 0 \text { for all } x \in \tau\right\} .
$$

If $\tau$ is the cone over a polytope $P$, then $\tau^{\vee}$ is the cone over $P^{*}$.

Theorem 4.3. There is a canonical isomorphism

$$
M_{k}(\tau) \cong \operatorname{or}(\tau) \otimes_{\mathbb{R}} \overline{\mathcal{L}\left(\tau^{\vee}\right)_{k}^{*}},
$$

where or $(\tau) \cong \mathbb{R}$ is the space of orientations of $\tau$.

There is an appealing special case of this result when $\operatorname{dim} \tau=2 k+1$. The degree $k$ part of $(22)$ gives an isomorphism $\overline{\mathcal{F}^{0}(\tau)}{ }_{k} \cong M_{k}(\tau)$. Thus there is a canonical dual pairing

$$
\overline{\mathcal{L}(\tau)}_{k} \otimes{\overline{\mathcal{L}\left(\tau^{\vee}\right)_{k}}}^{\rightarrow} \text { or }(\tau) \cong \operatorname{or}\left(\tau^{\vee}\right) .
$$


It is a pleasant exercise in linear algebra to construct such a pairing for $k=1$ using the descriptions of these vector spaces in Section 2.7. Giving an explicit description of such a pairing for $k=2$ in terms of the discussion in Section 2.8 seems to be much more difficult.

4.1. We sketch the proof of Theorem 4.3 using the toric Koszul duality constructed in [BL]. In that paper, it is shown that the derived category $D^{b}\left(\mathcal{A}_{\Delta}\right)$ of $\mathcal{A}_{\Delta}$-modules is a "mixed" version of the topological equivariant derived category $D_{T}^{b}\left(X_{\Delta}\right)$ of the toric variety $X_{\Delta}$. Roughly, this means that $D_{T}^{b}\left(X_{\Delta}\right)$ can be expressed as the derived category of modules over an associative ring $R$, and $D^{b}\left(\mathcal{A}_{\Delta}\right)$ is the derived category of graded modules over a graded version of $R$; these two categories are then related by a functor which forgets the grading. Thus $D^{b}\left(\mathcal{A}_{\Delta}\right)$ has two independent shift functors coming from shifting the grading in the complex and shifting the algebraic grading of the modules (note that the grading shift on graded $R$-modules is not the same as the grading shift on $\mathcal{A}_{\Delta}$-modules).

Every object in $D^{b}\left(\mathcal{A}_{\Delta}\right)$ is isomorphic to a complex of pure sheaves; pure sheaves and resolutions by them play a role similar to the role of injectives in other derived categories of sheaves. For instance, the resolution given by Theorem 4.1 gives an isomorphism $\mathcal{M} \stackrel{\sim}{\rightarrow} \mathcal{F}^{\bullet}$ in $D^{b}\left(\mathcal{A}_{\Delta}\right)$. The degree restrictions on the summands of the $\mathcal{F}^{i}$ says that this object $\mathcal{M}$ lies in the abelian subcategory $P\left(\mathcal{A}_{\Delta}\right) \subset D^{b}(\mathcal{A})$ of perverse objects, which corresponds to the abelian category of graded $R$-modules. The corresponding topological objects are equivariant perverse sheaves on $X_{\Delta}$. For instance, $\mathcal{M}$ represents the "Verma" or "standard" sheaf on $X_{\Delta}$ which is the extension by 0 of the rank one constant local system on the open orbit; this is perverse since the inclusion of the orbit is an affine map.

The simple objects in $P\left(\mathcal{A}_{\Delta}\right)$ are the sheaves ${ }_{\tau} \mathcal{L}$, taken with certain shifts which we ignore. The combinatorial IH sheaves in the complex $\mathcal{F}^{\bullet}$ are the simple constituents of the perverse object $\mathcal{M}$, and the "dumb" filtration obtained by truncating the complex gives the weight filtration on $\mathcal{M}$, which has semi-simple subquotients.

There are enough projectives in $P\left(\mathcal{A}_{\Delta}\right)$, so we can take a projective cover ${ }_{\tau} \mathcal{P}^{\bullet} \rightarrow{ }_{\tau} \mathcal{L}$. The total multiplicity space $M_{\bullet}(\tau)=\bigoplus_{i} M_{i}(\tau)$ is canonically isomorphic to the total Hom-space $\underline{\operatorname{Hom}}\left({ }_{\tau} \mathcal{P}^{\bullet}, \mathcal{M}\right)$ which takes all homomorphisms with all possible grading and degree shifts.

Now suppose that $\Delta=[\tau]$ for a full-dimensional rational cone $\tau$, and let $\Delta^{\vee}=\left[\tau^{\vee}\right]$. The main result of [BL] constructs an equivalence of triangulated categories

$$
K: D^{b}\left(\mathcal{A}_{\Delta}\right) \rightarrow D^{b}\left(L C_{\mathcal{F}}\left(X_{\Delta} \vee\right)\right)
$$


called the Koszul duality functor. The category on the right side is the derived category of sheaves on $X_{\Delta} \vee$ which are constructible with respect to the orbit stratification on $X_{\Delta} \vee$ and are endowed with an extra "mixed" structure.

Applying the Koszul functor $K$ sends ${ }_{\tau} \mathcal{P}$ to the intersection cohomology complex $I C^{\bullet}\left(X_{\Delta \vee} \vee\right.$, appropriately incarnated as an object in $D^{b}\left(L C_{\mathcal{F}}\left(X_{\Delta} \vee\right)\right)$. The image of $\mathcal{M}$ under $K$ is isomorphic to the point sheaf $i_{*} \mathbb{R}_{p}$ where $\{p\}$ is the the unique torus fixed point of $X_{\Delta \vee}$, and $i:\{p\} \rightarrow X_{\Delta \vee}$ is the inclusion.

Since $K$ is an equivalence of categories, it induces an isomorphism

$$
\underline{\operatorname{Hom}}\left({ }_{\tau} \mathcal{P}^{\bullet}, \mathcal{M}\right) \stackrel{K}{\longrightarrow} \underline{\operatorname{Hom}}\left(I C^{\bullet}\left(X_{\Delta} \vee\right), i_{*} \mathbb{R}_{p}\right) .
$$

The left side is identified with $M_{\bullet}(\tau)$, as we explained above, while the right side is the dual to the stalk intersection cohomology of $X_{\Delta} \vee$ by a standard adjunction. This essentially proves Theorem 4.3. The fact that the graded pieces on each side correspond follows from how the functor $K$ behaves under the shifts and twists from the mixed structure. The appearance of the orientation group in the theorem comes from pinning down $K(\mathcal{M})$ more precisely; it turns out to be canonically isomorphic to $i_{*}\left(\operatorname{or}(\tau)_{p}\right)$. This can be seen by noticing that $M_{0}(\tau) \cong \operatorname{or}(\tau)$, which follows from the proof of Lemma 4.4 below.

4.2. Proof of Theorem 4.1. We proceed by induction on the number of cones in the fan $\Delta$. If there is only one cone, then $\Delta=\{o\}$ and the result is obvious. If $\Delta$ has more than one cone, let $\sigma \in \Delta$ be a maximal cone, and let $\widetilde{\Delta}=\Delta \backslash\{\sigma\}$. Assume inductively that we have constructed the required resolution $0 \rightarrow \mathcal{M} \rightarrow$ $\widetilde{\mathcal{F}}^{0} \rightarrow \widetilde{\mathcal{F}}^{1} \rightarrow \cdots \rightarrow \widetilde{\mathcal{F}}^{s} \rightarrow 0, s=\operatorname{dim} \widetilde{\Delta}$ of sheaves on $\widetilde{\Delta}$. We will extend it to a resolution on $\Delta$.

For each $i$, let $\mathcal{E}^{i}$ be the sheaf on $\Delta$ which is the minimal pure extension of $\widetilde{\mathcal{F}}^{i}$. In other words, $\left.\mathcal{E}^{i}\right|_{\widetilde{\Delta}}=\widetilde{\mathcal{F}}^{i}$, and the restriction map makes $\mathcal{E}^{i}(\sigma)$ into the minimal free cover of $\mathcal{E}^{i}(\partial \sigma)$. Using the degree bounds from Theorem 2.6, it is easy to see that the maps $\widetilde{\mathcal{F}}^{i} \rightarrow \widetilde{\mathcal{F}}^{i+1}$ extend uniquely to maps $\mathcal{E}^{i} \rightarrow \mathcal{E}^{i+1}$ (see [BL, Theorem 6.6.2]). The resulting sequence of sheaves and maps

$$
0 \rightarrow \mathcal{M} \rightarrow \mathcal{E}^{0} \stackrel{d^{1}}{\longrightarrow} \mathcal{E}^{1} \stackrel{d^{2}}{\longrightarrow} \cdots \stackrel{d^{s}}{\longrightarrow} \mathcal{E}^{s}
$$

is almost the resolution we want, but the composition of successive maps may not be zero, and $d^{s}$ may not be surjective. We will add sheaves which are supported only on the cone $\sigma$ in order to recover the chain complex property.

Let $n=\operatorname{dim} \tau$, so $r=\operatorname{dim} \Delta=\max (n, s)$. For any $i \neq n$, let $\psi^{i}: \mathcal{E}^{i-1} \rightarrow \mathcal{E}^{i+1}$ be the composition $d^{i+1} \circ d^{i}$, and let $\mathcal{G}^{i}=\operatorname{Im} \psi^{i}$. Since $\left.\mathcal{E}^{\bullet}\right|_{\partial \sigma}$ is a complex by assumption, $\mathcal{G}^{i}$ has nonzero stalk only on $\sigma$. Using the degree restrictions on the simple constituents of the $\mathcal{E}^{j}$ together with Theorem 2.6, we see that $\mathcal{E}^{i-1}(\sigma)$ is a free $A_{\sigma}$-module generated in degrees $\leq \delta(i)$, where we put $\delta(j)=(n-j) / 2$. 
Further, $\mathcal{G}^{i}(\sigma)$ is contained in $\mathcal{E}^{i+1}(\sigma, \partial \sigma)$, which is a free $A_{\sigma}$-module generated in degrees $\geq \delta(i)$. It follows that $\mathcal{G}^{i}(\sigma)$ is a free $A_{\sigma}$-module generated in degree exactly $\delta(i)$. In particular, $\mathcal{G}^{i}=0$ if $i$ and $n$ have different parity or if $i \geq n$.

Let $\mathcal{G}^{n}$ be the cokernel of $d^{n-1}$ on $[\sigma]$, extended by zero to the other cones; it is supported only on $\sigma$. Since $\mathcal{E}^{n-2}(\sigma)$ and $\mathcal{E}^{n-1}(\sigma)$ are free $A_{\sigma}$-modules generated in degree 0 , so is $\mathcal{G}^{n}(\sigma)$.

Define $\mathcal{F}^{i}=\mathcal{E}^{i} \oplus \mathcal{G}^{i}$. We make these sheaves into a complex by defining the boundary maps to be

$$
\cdots \rightarrow \mathcal{E}^{i-1} \stackrel{\left[{ }_{-\psi^{i}}^{i}\right]}{\longrightarrow} \mathcal{E}^{i} \oplus \mathcal{G}^{i} \stackrel{\left[d^{i+1} \iota\right]}{\longrightarrow} \mathcal{E}^{i+1} \rightarrow \cdots
$$

for any $i \neq n$ with $i-n$ even, where $\iota: \mathcal{G}^{i} \rightarrow \mathcal{E}^{i+1}$ is the inclusion, and defining the boundary maps at the $n$th position to be

$$
\cdots \rightarrow \mathcal{E}^{n-1} \stackrel{\left[\begin{array}{l}
d^{n} \\
p
\end{array}\right]}{\longrightarrow} \mathcal{E}^{n} \oplus \mathcal{G}^{n} \stackrel{\left[d^{n+1} 0\right]}{\longrightarrow} \mathcal{E}^{n+1} \rightarrow \cdots,
$$

where $p: \mathcal{E}^{n-1} \rightarrow$ coker $d^{n-1}$ is the natural projection, extended by zero.

It is easy to check that this is a complex. To see that it is a resolution of $\mathcal{M}$, we need to show that $\mathcal{F}^{\bullet}(\sigma)$ is an exact sequence, since $\mathcal{M}(\sigma)=0$. Since $\mathcal{F}^{\bullet}(\sigma)$ is a complex of free $A_{\sigma}$-modules, it will be exact if and only if the reduced complex $\overline{\mathcal{F}^{\bullet}(\sigma)}$ is an exact sequence of graded vector spaces. The degree $k$ part $\overline{\mathcal{F}}^{i}(\sigma)_{k}$ vanishes if $i>n-2 k$, and if $i=n-2 k$ (so $\delta(i)=k$ ), the boundary $\overline{\mathcal{F}}^{i-1}(\sigma)_{k} \rightarrow \overline{\mathcal{F}}^{i}(\sigma)_{k}=\mathcal{G}^{i}\left(\sigma_{k}\right)$ is surjective by construction. So we only need to check exactness at $i<n-2 k$.

Lemma 4.4. $H^{i}\left(\mathcal{F}^{\bullet}(\partial \sigma)\right)=0$ if $i \neq n-1$, and $H^{n-1}\left(\mathcal{F}^{\bullet}(\partial \sigma)\right)$ is isomorphic to $\mathbb{R}$, placed in degree zero.

Assuming this for the moment, consider the short exact sequence

$$
0 \rightarrow \mathcal{F}^{\bullet}(\sigma, \partial \sigma) \rightarrow \mathcal{F}^{\bullet}(\sigma) \rightarrow \mathcal{F}^{\bullet}(\partial \sigma) \rightarrow 0
$$

of chain complexes. It induces a long exact cohomology sequence

$$
\cdots \rightarrow H^{i}\left(\overline{\mathcal{F}^{\bullet}(\sigma, \partial \sigma)}\right) \rightarrow H^{i}\left(\overline{\mathcal{F}^{\bullet}(\sigma)}\right) \rightarrow H^{i}\left(\mathcal{F}^{\bullet}(\partial \sigma) \stackrel{L}{\otimes} A_{\sigma} \mathbb{R}\right) \rightarrow \cdots,
$$

where the third term is the cohomology of a derived functor, since $\mathcal{F}^{\bullet}(\partial \sigma)$ is not a complex of free modules. The first term vanishes in degrees $<\delta(i)$, the second vanishes in degrees $>\delta(i)$, and the last term is nonzero only in degree $n-1-i$, since by the lemma $\mathcal{F}^{\bullet}(\partial \sigma)$ has a free resolution by a Koszul complex.

Combined with our previous observations, this implies that $H^{i}\left(\overline{\mathcal{F}^{\bullet}(\sigma)}\right)_{k}=0$ except possibly when $k=0$ and $i=n-1$. But the complexes $\mathcal{F}^{\bullet}(\sigma)_{0}$ and $\mathcal{F}^{\bullet}(\partial \sigma)_{0}$ are identical except that $\mathcal{F}^{k}(\sigma)_{0}=\mathbb{R}$ while $\mathcal{F}^{k}(\partial \sigma)_{0}=0$. Lemma 4.4 thus implies 
that the Euler characteristic of $\mathcal{F}^{\bullet}(\sigma)_{0}$ is zero, so the last cohomology group also vanishes, completing the construction of our resolution.

The uniqueness of $\mathcal{F}^{\bullet}$ is easily proved by following the same induction. Any automorphism of the complex $\widetilde{\mathcal{F}} \bullet$ extends uniquely to an automorphism of $\mathcal{E} \bullet$, since the sheaves ${ }_{\tau} \mathcal{L}$ themselves have only scalar automorphisms. This extends uniquely to an automorphism of $\mathcal{F}^{\bullet}$, since the maps $\psi^{i}: \mathcal{E}^{i-1} \rightarrow \mathcal{G}^{i}$ and $p: \mathcal{E}^{n-1} \rightarrow$ $\mathcal{G}^{n}$ are surjective.

Proof of Lemma 4.4. The global sections of an exact sequence of flabby sheaves is exact, so the positive degree parts of the sequence $\mathcal{F}^{\bullet}(\partial \sigma)$ are exact sequences of vector spaces.

In degree zero, it is easy to see by induction that $\mathcal{F}_{0}^{i}=\bigoplus_{\operatorname{dim} \tau=i} \underline{\mathbb{R}}_{\mathrm{st}(\tau)}$, and that the component of the boundary map $\underline{\mathbb{R}}_{\mathrm{st}(\tau)} \rightarrow \mathbb{R}_{\mathrm{st}(\rho)}$ for $\operatorname{dim} \rho=\operatorname{dim} \tau+1$ is non-zero if and only if $\tau$ is a face of $\rho$. Taking global sections, the complex $\mathcal{F}^{\bullet}(\partial \sigma)_{0}$ is isomorphic to the augmented cellular chain complex of the regular cell complex obtained by intersecting the fan $\partial \sigma$ with a sphere centered at the origin. This cell complex is a $(n-1)$-sphere.

\section{APPENDiX: TWO RESUlTS OF KALAi}

With his kind permission, we present Kalai's previously unpublished proofs of two consequences of his monotonicity conjecture (Theorem 1.2). The first relates the $g$-numbers of a polytope $P$ and its polar $P^{*}$.

Theorem 4.5 (Kalai). If $g_{k}(P)=0$, then $g_{k}\left(P^{*}\right)=0$.

Proof. Use induction on $k$ and $d$. The case $k=0$ is trivial, since $g_{0}(P)=1$ for all polytopes $P$. Similarly, if $d<2 r$, then $g_{r}(P)=g_{r}\left(P^{*}\right)=0$, and there is nothing to prove.

Otherwise, assume the result holds when $k<r$ or $k=r$ and $\operatorname{dim} P<d$. The degree $r$ term of Stanley's formula (20) gives

$$
\sum_{\emptyset \leq F \leq P} \sum_{i+j=r}(-1)^{\operatorname{dim} F} g_{i}\left(F^{*}\right) g_{j}(P / F)=0 .
$$

If $g_{k}(P)=0$, then Theorem 1.2 implies that $g_{i}(F) g_{j}(P / F)=0$ for all $i+j=k$ and all faces $F$ of $P$. By the inductive hypothesis this means that all the terms of $(24)$ vanish except for $g_{k}\left(P^{*}\right) g_{0}(P / P)=g_{k}\left(P^{*}\right)$.

The next result is Theorem 1.4 from Section 1, which says that $g_{k}(P)=0$ implies $g_{k+1}(P)=0$. This follows from Theorem 1.2 and the following identity. 
Proposition 4.6 (Kalai). For any $d$-polytope $P$ and $0 \leq k \leq d / 2-1$,

$$
(k+1) g_{k+1}(P)+(d-k+1) g_{k}(P)=\sum_{i=0}^{k}(i+1) \sum_{\substack{F \leq P \\ \operatorname{dim} F=2 i}} g_{i}(F) g_{k-i}(P / F) .
$$

This generalizes the identity for simplicial polytopes

$$
\sum_{v} g_{k}(P / v)=(d-k+1) g_{k}(P)+(k+1) g_{k+1}(P)
$$

(summing over all vertices of $P$ ), which is used in the proof of the upper bound theorem for simplicial polytopes [McM1]. The special case $d=2 k+1$ was previously obtained by Stenson [Ste].

We will use Kalai's convolution notation: if $\phi_{1}$ and $\phi_{2}$ are linear expressions in the flag numbers of $d_{1}$ and $d_{2}$-polytopes, respectively, this gives an invariant $\phi * \psi$ of $\left(d_{1}+d_{2}+1\right)$-polytopes by

$$
\phi * \psi(P)=\sum_{\substack{F \leq P \\ \operatorname{dim} F=d_{1}}} \phi(F) \psi(P / F) ;
$$

it is again a linear combination of flag numbers.

Define $\tilde{g}_{k}(P)=h_{k}(P)-h_{k-1}(P)$ for all $k$, not just $k \leq d / 2$, so by the DehnSommerville relations we have

$$
\tilde{g}_{k}(P)= \begin{cases}g_{k}(P) & k \leq d / 2 \\ -g_{d-k+1}(P) & k \geq d / 2+1 \\ 0 & k=(d+1) / 2(d \text { odd }) .\end{cases}
$$

Let $\tilde{g}_{k}^{d}$ denote the invariant $\tilde{g}_{k}$ applied to $d$-polytopes. It will be more convenient for induction to prove the following generalization of Proposition 4.6.

$$
(k+1) \tilde{g}_{k+1}(P)+(d-k+1) \tilde{g}_{k}(P)=\sum_{i=0}^{k}(i+1) \tilde{g}_{i}^{2 i} * \tilde{g}_{k-i}^{d-2 i-1}(P) .
$$

Since this formula is linear in the flag vectors, it is enough to check it on a basis of polytopes, i.e. a collection of polytopes whose flag $f$-vectors are a basis for the linear span of all flag $f$-vectors. Using the basis given in [BayBi], this amounts to checking (25) when $d=0$, and inductively showing that if it holds for all $d-1$ polytopes $Q$, then it holds for the cone $C Q$ and the bipyramid $B Q$.

The case when $d$ is even and $k=d / 2$ is immediate, since the left-hand side is $(k+1)\left(-g_{k}(P)+g_{k}(P)\right)=0$, while each term on the right contains $\tilde{g}_{k-i}^{2(k-i)-1} \equiv 0$. In particular, the base case $d=0$ is established. 
Next, suppose that $d>0$ and $P=C Q$ for a $d-1$ polytope $Q$. By the symmetry of the $\tilde{g}_{k}$ 's it is enough to establish (25) for $0 \leq k<d / 2$. We have $g_{k}(P)=g_{k}(Q)$ for all $k$ (see [Kal2]), which implies that $\tilde{g}_{k}(P)=\tilde{g}_{k}(Q)$ if $k \leq d / 2$. Thus if $k \leq d / 2-1$ the left side of $(25)$ becomes

$$
(k+1) \tilde{g}_{k+1}(Q)+(d-k+1) \tilde{g}_{k}(Q)=\tilde{g}_{k}(Q)+\sum_{i=0}^{k}(i+1) g_{i}^{2 i} * \tilde{g}_{k-i}^{d-2 i-2}(Q),
$$

by the inductive hypothesis. On the other hand, the nonempty faces of $C Q$ are either nonempty faces of $Q$ or cones over faces (possibly empty). Since $g_{k}(C F)=$ 0 if $\operatorname{dim}(C F)=d$, the only $2 i$-faces of $P$ for which $g_{i} \neq 0$ are the apex $C \emptyset$ and faces of $Q$. Thus the right side of (25) becomes

$$
\tilde{g}_{k}(Q)+\sum_{i=0}^{k}(i+1) g_{i}^{2 i} * \tilde{g}_{k-i}^{d-2 i-2}(Q)
$$

as required.

If $d$ is odd and $k=(d-1) / 2$, we have $\tilde{g}_{k}(P)=\tilde{g}_{k}(Q)$, but $\tilde{g}_{k+1}(P)=0$, so the left side of $(25)$ is just $(k+2) \tilde{g}_{k}(Q)$. Since for every face $F$ of $P$ other than the apex $C \emptyset$ and the base $Q$ either $F$ or $P / F$ is a cone over a nonempty polytope, the right side is

$\sum_{i=0}^{k}(i+1) g_{i}^{2 i} * \tilde{g}_{k-i}^{2(k-i)}(P)=g_{0}(C \emptyset) g_{k}(Q)+(k+1) g_{k}(Q) g_{0}(P / Q)=(k+2) g_{k}(Q)$.

Therefore (25) holds for $P=C Q$.

Now suppose that $P=B Q$, the bipyramid over a $(d-1)$-polytope $Q$. Then $h_{P}(t)=(t+1) h_{Q}(t)$ (see [Kal2]), so we have

$$
\tilde{g}_{k}(P)=\tilde{g}_{k}(Q)+\tilde{g}_{k-1}(Q)
$$

for all $0 \leq k \leq d+1$. Thus the left side of our identity is

$$
\begin{aligned}
& (k+1)\left(\tilde{g}_{k+1}(Q)+\tilde{g}_{k}(Q)\right)+(d-k+1)\left(\tilde{g}_{k}(Q)+\tilde{g}_{k-1}(Q)\right) \\
= & 2 \tilde{g}_{k}(Q)+\sum_{i=0}^{k}(i+1) g_{i}^{2 i} * \tilde{g}_{k-i}^{d-2 i-2}(Q)+\sum_{i=0}^{k-1}(i+1) g_{i}^{2 i} * \tilde{g}_{k-1-i}^{d-2 i-2}(Q),
\end{aligned}
$$

by the inductive hypothesis.

On the other hand, faces of $P=B Q$ are either faces of $Q$ other than $Q$ itself or cones over faces of $Q$ (possibly empty). The only $2 i$-faces $F \leq B Q$ for which $g_{i}^{2 i}(F)$ can be nonzero are the two apexes, for which $g_{0}(F) \tilde{g}_{k}(B Q / F)=\tilde{g}_{k}(Q)$, and faces $F \leq Q, F \neq Q$, for which

$$
g_{i}(F) g_{k-i}((B Q) / F)=g_{i}(F) \tilde{g}_{k-i}(B(Q / F))=g_{i}(F)\left[\tilde{g}_{k-i}(Q / F)+\tilde{g}_{k-i-1}(Q / F)\right] \text {. }
$$

Substituting these into the right side of (25) gives (26). 


\section{REFERENCES}

[BBFK1] G. Barthel, J.-P. Brasselet, K.-H. Fieseler, and L. Kaup. Equivariant intersection cohomology of toric varieties. In Algebraic geometry: Hirzebruch 70 (Warsaw, 1998), volume 241 of Contemp. Math., pages 45-68. Amer. Math. Soc., Providence, RI, 1999.

[BBFK2] _ Combinatorial intersection cohomology for fans. Tohoku Math. J. (2), $54(1): 1-41,2002$.

[BBFK3] Combinatorial duality and intersection product: a direct approach. Tohoku Math. J. (2), 57(2):273-292, 2005.

[BBFK4] Hodge-Riemann relations for polytopes — a geometric approach. preprint arXiv:math.AG/0602411, 29pp.

[BaBo] V. V. Batyrev and L. Borisov. Mirror duality and string-theoretic Hodge numbers. Invent. Math. 126(1):183-206, 1996.

[Bay1] M. M. Bayer. An upper bound theorem for rational polytopes. J. Combin. Theory Ser. A, 83(1):141-145, 1998.

[Bay2] Shelling and the $h$-vector of the (extra)ordinary polytope. In Combinatorial and Computational Geometry, number 52 in MSRI Publications, pages 97-120. Cambridge University Press, 2005.

[BayBi] M. M. Bayer and L. J. Billera. Generalized Dehn-Sommerville relations for polytopes, spheres and Eulerian partially ordered sets. Invent. Math., 79(1):143-157, 1985.

[BayE] M. M. Bayer and R. Ehrenborg. The toric $h$-vectors of partially ordered sets. Trans. Amer. Math. Soc., 352(10):4515-4531 (electronic), 2000.

[BeL] J. Bernstein and V. Lunts. Equivariant sheaves and functors, volume 1578 of Lecture Notes in Mathematics. Springer-Verlag, Berlin, 1994.

[BiL] L. J. Billera and C. W. Lee. A proof of the sufficiency of McMullen's conditions for $f$-vectors of simplicial convex polytopes. J. Combin. Theory Ser. A, 31(3):237-255, 1981.

[BoM] L. Borisov and A. R. Mavlyutov. String cohomology of Calabi-Yau hypersurfaces via Mirror Symmetry. Adv. Math. 180(1):355-390, 2003.

[B] T. Braden. Hyperbolic localization of intersection cohomology. Transform. Groups, 8(3):209-216, 2003.

[BL] T. Braden and V. Lunts. Equivariant-constructible Koszul duality for dual toric varieties. Adv. Math. 201(2):408-453, 2006.

[BM] T. Braden and R. MacPherson. Intersection homology of toric varieties and a conjecture of Kalai. Comment. Math. Helv., 74(3):442-455, 1999.

[BM2] 근 from moment graphs to intersection cohomology. Math. Ann., 321(3):533$551,2001$.

[BrL1] P. Bressler and V. A. Lunts. Intersection cohomology on nonrational polytopes. Compositio Math., 135(3):245-278, 2003.

[BrL2] Hard Lefschetz theorem and Hodge-Riemann relations for intersection cohomology of nonrational polytopes. Indiana Univ. Math. J., 54(1):263-307, 2005.

[Bri] M. Brion. The structure of the polytope algebra. Tohoku Math. J. (2), 49(1):1-32, 1997.

[BruM] H. Bruggesser and P. Mani. Shellable decompositions of cells and spheres. Math. Scand., 29:197-205 (1972), 1971.

[Bry] J.-L. Brylinski. Equivariant intersection cohomology. In Kazhdan-Lusztig theory and related topics (Chicago, IL, 1989), volume 139 of Contemp. Math., pages 5-32. Amer. Math. Soc., Providence, RI, 1992.

[C1] R. Connelly. A counterexample to the rigidity conjecture for polyhedra. Inst. Hautes Études Sci. Publ. Math., (47):333-338, 1977. 
[C2] , A flexible sphere. Math. Intelligencer, 1(3):130-131, 1978/79.

[DL] J. Denef and F. Loeser. Weights of exponential sums, intersection cohomology, and Newton polyhedra. Invent. Math., 106(2):275-294, 1991.

[E] R. Ehrenborg. Lifting inequalities for polytopes. Adv. Math., 193(1):205-222, 2005.

[FP] M. Fedorchuk and I. Pak. Rigidity and polynomial invariants of convex polytopes, Duke Math. J., 129(2):371-404, 2005.

[Fieb] P. Fiebig. Kazhdan-Lusztig combinatorics via sheaves on Bruhat graphs. preprint arXiv:math.RT/0512311.

[Fies] K.-H. Fieseler. Rational intersection cohomology of projective toric varieties. J. Reine Angew. Math., 413:88-98, 1991.

[FuS] W. Fulton and B. Sturmfels. Intersection theory on toric varieties. Topology, 36(2):335-353, 1997.

[GKM] M. Goresky, R. Kottwitz, and R. MacPherson. Equivariant cohomology, Koszul duality, and the localization theorem. Invent. Math., 131(1):25-83, 1998.

[GZ1] V. Guillemin and C. Zara. Equivariant de Rham theory and graphs. Asian J. Math., 3(1):49-76, 1999.

[GZ2] , 1-skeleta, Betti numbers, and equivariant cohomology. Duke Math. J., 107(2):283-349, 2001.

[GZ3] , G-actions on graphs. Internat. Math. Res. Notices, (10):519-542, 2001.

[GZ4] The existence of generating families for the cohomology ring of a graph. Adv. Math., 174(1):115-153, 2003.

[J] R. Joshua. Vanishing of odd-dimensional intersection cohomology. Math. Z., 195(2):239-253, 1987.

[Kal1] G. Kalai. Rigidity and the lower bound theorem. I. Invent. Math., 88(1):125-151, 1987.

[Kal2] - A new basis of polytopes. J. Combin. Theory Ser. A, 49(2):191-209, 1988.

[Kal3] Some aspects of the combinatorial theory of convex polytopes. In Polytopes: abstract, convex and computational (Scarborough, ON, 1993), volume 440 of NATO Adv. Sci. Inst. Ser. C Math. Phys. Sci., pages 205-229. Kluwer Acad. Publ., Dordrecht, 1994.

[Kar1] Hard Lefschetz theorem for nonrational polytopes. Invent. Math., 157(2):419447, 2004.

[Kar2] K. Karu. Lefschetz decomposition and the cd-index of fans. arXiv:math.AG/0509220.

[Kar3] _ The cd-index of fans and lattices. arXiv:math.AG/0410513.

[Kir] F. Kirwan. Intersection homology and torus actions. J. Amer. Math. Soc., 1(2):385400, 1988.

[L] C. W. Lee. Some recent results on convex polytopes. In Mathematical developments arising from linear programming (Brunswick, ME, 1988), volume 114 of Contemp. Math., pages 3-19. Amer. Math. Soc., Providence, RI, 1990.

[L2] Generalized stress and motions. In Polytopes: abstract, convex and computational (Scarborough, ON, 1993), volume 440 of NATO Adv. Sci. Inst. Ser. C Math. Phys. Sci., pages 249-271. Kluwer Acad. Publ., Dordrecht, 1994.

[McC] M. McConnell. The rational homology of toric varieties is not a combinatorial invariant. Proc. Amer. Math. Soc., 105(4):986-991, 1989.

[McM1] P. McMullen. The maximum numbers of faces of a convex polytope. Mathematika, $17: 179-184,1970$.

[McM2] _ On simple polytopes. Invent. Math., 113(2):419-444, 1993.

[P] I. Pak, A short proof of rigidity of convex polytopes, preprint (2005); to appear in Siberian J. Mathematics. 
[S] I. K. Sabitov. The volume as a metric invariant of polyhedra. Discrete Comput. Geom., 20(4):405-425, 1998.

[St1] The number of faces of a simplicial convex polytope. Adv. in Math., 35(3):236-238, 1980.

[St2] R. Stanley. Generalized $H$-vectors, intersection cohomology of toric varieties, and related results. In Commutative algebra and combinatorics (Kyoto, 1985), volume 11 of Adv. Stud. Pure Math., pages 187-213. North-Holland, Amsterdam, 1987.

[St3] Subdivisions and local $h$-vectors. J. Amer. Math. Soc., 5(4):805-851, 1992.

[Ste] C. Stenson. Relationships among flag $f$-vector inequalities for polytopes. Discrete Comput. Geom., 31(2):257-273, 2004.

[TWW1] T.-S. Tay, N. White, and W. Whiteley. Skeletal rigidity of simplicial complexes. I. European J. Combin., 16(4):381-403, 1995.

[TWW2] Skeletal rigidity of simplicial complexes. II. European J. Combin., 16(5):503523, 1995.

[Ti] V. A. Timorin. An analogue of the Hodge-Riemann relations for simple convex polyhedra. Uspekhi Mat. Nauk, 54(2(326)):113-162, 1999.

[W] W. Whiteley. Infinitesimally rigid polyhedra. I. Statics of frameworks. Trans. Amer. Math. Soc., 285(2):431-465, 1984.

[Z] G. M. Ziegler. Lectures on polytopes, volume 152 of Graduate Texts in Mathematics. Springer-Verlag, New York, 1995.

\section{Tom Braden}

University of Massachusetts, Amherst

E-mail: braden@math.umass.edu 